\title{
Sur une majoration explicite pour un degré d'isogénie liant deux courbes elliptiques
}

\author{
par \\ Federico Pellarin (Caen)
}

1. Introduction, énoncés des résultats. Dans ce texte, $K$ désigne un corps de nombres avec un plongement $K \rightarrow \mathbb{C}$ fixé une fois pour toutes dans le corps $\mathbb{C}$ des nombres complexes, et $d=\max \{2,[K: \mathbb{Q}]\}$. Dans toute la suite on note $E$ et $E^{\sharp}$ deux courbes elliptiques définies sur $K$ (notation : $\left.\left.E\right|_{K},\left.E^{\sharp}\right|_{K}\right)$, c'est-à-dire munies de modèles de Weierstrass

$$
\begin{array}{ll}
E: & u^{2}=4 x^{3}-g_{2} x-g_{3}, \\
E^{\sharp}: & u^{2}=4 x^{3}-g_{2}^{\sharp} x-g_{3}^{\sharp},
\end{array}
$$

avec $g_{2}, g_{3}, g_{2}^{\sharp}, g_{3}^{\sharp} \in K$.

Nous associons à $E$ une hauteur naïve dépendant du choix du modèle de Weierstrass, $h(E)=\max \left\{1, h\left(j_{E}\right)\right\}+\max \left\{1, h\left(1: g_{2}: g_{3}\right)\right\}$, où $j_{E} \in K$ est le $j$-invariant de $E$ (cf. chapitre 3 de [Lan]). Notons aussi $h_{\mathcal{F}}(E)$ la hauteur modulaire semi-stable de Faltings (cf. [Fal] pour les propriétés de base).

ThÉORÈme 1 (Hauteurs naïves). Soit $K$ un corps de nombres, posons $d=\max \{2,[K: \mathbb{Q}]\}$, et soit $E$ une courbe elliptique définie sur $K$. Pour toute courbe elliptique $E^{\sharp}$ définie sur $K$ et isogène à $E$ il existe une isogénie $\psi: E \rightarrow E^{\sharp}$ dont le degré $N$ satisfait la majoration

$$
N \leq 4.2 \cdot 10^{61} d^{4} \max \{1, \log d\}^{2} h(E)^{2} .
$$

Nous donnons aussi une version de ce théorème en termes de hauteurs semi-stables.

ThÉORÈme 2 (Hauteurs semi-stables). Sous les hypothèses du théorème 1 il existe une isogénie $\psi: E \rightarrow E^{\sharp}$ dont le degré $N$ satisfait la majoration

$$
N \leq 10^{78} d^{4} \max \{1, \log d\}^{2} \max \left\{1, h_{\mathcal{F}}(E)\right\}^{2} .
$$

2000 Mathematics Subject Classification: Primary 11J89. 
1.1. Motivations. Nos deux théorèmes 1 et 2 s'inspirent directement du résultat principal de l'article de Masser et Wüstholz [Ma-Wü4]. Pour aboutir à nos estimations numériques explicites, nous avons largement puisé dans les travaux de David, spécialement [Da]. Les techniques utilisées par ces auteurs remontent aux travaux de Baker dans les groupes linéaires commutatifs et sur les minorations effectives de formes linéaires de logarithmes de nombres algébriques, vus à travers le prisme du programme de Lang, visant à généraliser ces travaux aux groupes algébriques commutatifs définis sur les corps de nombres.

Une place très importante est occupée dans ce type de problèmes par les lemmes de zéros dans les groupes algébriques commutatifs, comme ceux de Denis et Philippon ([Den], [Phi1] et [Phi2]).

Le lien avec les formes linéaires de logarithmes elliptiques apparaît assez naturellement en observant qu'une isogénie liant deux courbes elliptiques induit des relations linéaires non nulles entre périodes à coefficients qui sont essentiellement des entiers rationnels, et des relations algébriques entre fonctions de Weierstrass. Il s'agit de montrer que si les coefficients de toutes les relations linéaires possibles liant les périodes de deux courbes elliptiques isogènes étaient "trop grands", alors on aurait une contradiction avec l'existence d'une certaine relation algébrique de "petit degré" entre les fonctions de Weierstrass associées aux modèles des deux courbes elliptiques.

Dans [Ma-Wü4] Masser et Wüstholz ont démontré, sous les hypothèses du théorème 1 , qu'il existe une isogénie dont le degré est majoré par $c_{1} h(E)^{4}$ : la constante $c_{1}$ est "effective et dépend uniquement du degré $[K: \mathbb{Q}]$ ", mais reste imprécisée.

Cet article [Ma-Wü4] a inauguré une série de résultats plus généraux sur les variétés abéliennes, dont nous mentionnons ici seulement [Ma-Wü1] et [Ma-Wü2]. Le but final est de montrer comment les techniques transcendantes peuvent s'appliquer pour prouver, entre autres, des versions "plus effectives" des conjectures de Tate et Shafarevich.

Notre direction de recherche a été différente : nous nous sommes limité aux courbes elliptiques définies sur les corps de nombres mais nous avons cherché une meilleure dépendance en la quantité $h(E)$ dans le résultat final, tout en donnant des résultats numériquement explicites.

David obtient, comme corollaire à son théorème principal (corollaire 2.2, p. 11 de $[\mathrm{Da}])$, la majoration suivante pour le degré minimal d'isogénie : $N \leq 10^{160}[K: \mathbb{Q}]^{20} h(E)^{10}$ (mêmes hypothèses que le théorème $1\left(^{1}\right)$ ). Cette majoration est totalement explicite, mais la dépendance en $h(E)$ est moins bonne que la nôtre, ou celle donnée par Masser et Wüstholz, puisque David

$\left({ }^{1}\right)$ Rappelons que David utilise dans [Da] une autre hauteur naïve $: \max \left\{h\left(1: g_{2}:\right.\right.$ $\left.\left.g_{3}\right), h\left(j_{E}\right)\right\}$. 
travaille avec une seule forme linéaire. Nous utiliserons cependant plusieurs astuces introduites par David, pour fournir des constantes explicites relativement bonnes.

Soulignons que nos théorèmes 1 et 2 s'appliquent, en utilisant des arguments simples déjà décrits par Masser et Wüstholz, pour fournir une version explicite du théorème d'irréductibilité de Serre, et une version explicite du théorème de finitude de Shafarevich : on pourra utiliser les techniques de [Ma-Wü3] pour démontrer les corollaires suivants du théorème 2.

Corollaire 1. Soit $K$ un corps de nombres, soit $d=\max \{2,[K: \mathbb{Q}]\}$ et soit $\left.E\right|_{K}$ une courbe elliptique sans multiplication complexe sur $\bar{K}$. Soit $\ell$ un nombre premier et $\varrho_{\ell}: \operatorname{Gal}(\bar{K} \mid K) \rightarrow \operatorname{Aut}(E[\ell])$ la représentation (de Serre, cf. [Se]) sur les points de $\ell$-torsion de E. Si

$$
\ell \geq 10^{78} d^{4} \max \{1, \log d\}^{2} \max \left\{1, h_{\mathcal{F}}(E)\right\}^{2}
$$

alors $\varrho_{\ell}$ est irréductible.

Corollaire 2. Sous les hypothèses du corollaire précédent, il existe au plus $10^{156} d^{8} \max \{1, \log d\}^{4} \max \left\{1, h_{\mathcal{F}}(E)\right\}^{4}$ classes de K-isomorphisme de courbes elliptiques $E^{\sharp}$ définies sur $K$ et isogènes à $E$.

Sous les hypothèses du corollaire 1 , en supposant que $K$ admet une place réelle, on peut calculer une constante numérique $c_{2}>0$ telle que pour tout nombre premier $\ell>c_{2} d^{4} \max \{1, \log d\}^{2} \max \left\{1, h_{\mathcal{F}}(E)\right\}^{2}$, l'image de $\varrho_{\ell}$ contient une copie du groupe linéaire spécial $\mathrm{SL}_{2}\left(\mathbb{F}_{\ell}\right)$ (ici $\mathbb{F}_{\ell}$ est le corps à $\ell$ éléments).

On peut parvenir à cette même conclusion si l'on suppose que la courbe elliptique $E$ satisfaisant les hypothèses du corollaire 1 ait de plus son invariant modulaire $j_{E}$ non entier. Nous ne calculons pas la constante $c_{2}$ dans ce texte car les arguments de [Ma-Wü3] permettent de le faire sans difficulté.

Remarques. L'exposant 2 de $h(E)$ dans l'inégalité (2) avait été prévu par Bertrand dans [Be], p. 123.

En adaptant la construction de Masser et Wüstholz (pp. 455-456 de [Ma-Wü2]) on peut montrer que, sous les hypothèses du théorème 1 , les arguments utilisés dans la démonstration de leur théorème p. 408 ne sont pas suffisants pour construire une isogénie $\psi: E \rightarrow E^{\sharp}$ dont le degré $N$ satisfait une majoration de type $N \leq c_{3}(d) h(E)^{2-\varepsilon}$ avec $0<\varepsilon \leq 2$ pour une constante $c_{3}(d)>0$ ne dépendant que de $d$.

1.2. Structure de la preuve. Le résultat principal de ce texte est le théorème 1 et le théorème 2 en découle directement. Décrivons d'abord les traits essentiels de la preuve du théorème 1 ; nous évoquons aussi le théorème p. 408 de [Ma-Wü2] et nous le comparons avec le théorème principal dans [Ma-Wü4]. 
Soient $E$ et $E^{\sharp}$ deux courbes elliptiques définies sur un corps de nombres $K$, et supposons-les isogènes. Fixons aussi un plongement $\sigma$ de $\bar{K}$ dans $\mathbb{C}$. Soient $\omega_{1}, \omega_{2}$ et $\omega_{1}^{\sharp}, \omega_{2}^{\sharp}$ des périodes fondamentales des réseaux de périodes de $E$ et $E^{\sharp}$ respectivement.

Nous avons une période non nulle $\omega=\left(\omega_{1}, \omega_{2}, \omega_{1}^{\sharp}, \omega_{2}^{\sharp}\right)$ dans le réseau des périodes de la variété abélienne $G=E \times E \times E^{\sharp} \times E^{\sharp}$ que nous plongeons dans un espace projectif $\mathbb{P}_{\eta}(\mathbb{C})$ au moyen des modèles de Weierstrass et des plongements de Segre itérés.

Soit $T_{\mathbb{C}}=T(G) \otimes_{\sigma} \mathbb{C}$ l'espace vectoriel complexe tangent à l'origine de $G$ et soit $\Delta=\mathbb{C} \omega$ la droite engendrée par $\omega$ dans $T_{\mathbb{C}}$ (dans ce texte nous appellerons $\Delta$ la droite de Baker).

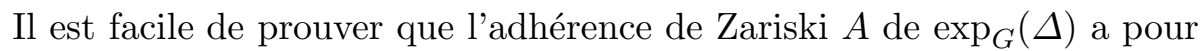
dimension 2 si et seulement si $E$ n'a pas de multiplication complexe ; supposons ici que $E$ n'a pas de multiplication complexe.

Le théorème p. 408 de [Ma-Wü2] implique que le degré de $A$ est majoré par $c(d) h(E)^{\kappa}$, où $c(d)$ est une constante dépendant de manière explicite de $d$, et $\kappa$ est un entier naturel. Mais en appliquant des techniques de preuve similaires à celles de [Ma-Wü2] on peut plus simplement prouver qu'il existe une sous-variété abélienne $\mathfrak{H}$ de $G$ dont le degré est majoré par une constante plus petite $c(d) h(E)^{2}$, et satisfaisant une des conditions $(*)$ suivantes : $\mathfrak{H}$ est de codimension 1 dans $A, \mathfrak{H}=A$ ou $A$ est de codimension 1 dans $\mathfrak{H}$.

Les conditions $(*)$ sur $\mathfrak{H}$ sont en général suffisantes pour les estimations de degré d'isogénie et l'exposant 2 que l'on obtient ainsi est meilleur que l'exposant $\kappa$ que l'on obtiendrait plus haut.

Le lemme de Bézout et le principe de Goursat-Kolchin-Ribet (lemme 2.2 dans ce texte) impliquent

$$
N \leq c(d) h(E)^{4} .
$$

Cette méthode est essentiellement celle ad hoc employée dans [Ma-Wü4].

Pour améliorer la dépendance en la hauteur de l'estimation ci-dessus il faut analyser avec plus de soin la partie transcendante de la démonstration donnée dans [Ma-Wü4] ; oublions pour le moment la dépendance en $d$. Nous allons justifier brièvement pourquoi Masser et Wüstholz obtiennent un exposant 4 dans (4).

Rappelons que dans [Ma-Wü4] il s'agit, tenant compte du lemme de multiplicités, de construire un polynôme $P$ de degré $D$ dans l'anneau des coordonnées de $\mathbb{P}_{\eta}$ dont la restriction à $G$ soit non nulle et s'annulant à un ordre $\geq T$ le long de l'espace tangent à l'origine de $A$ sur un ensemble donné de points de torsion. Il n'est pas difficile de s'aperçevoir que cette multiplicités d'annulation doit satisfaire $T \geq c(d) D^{2}$ pour une constante $c(d)$ ne dépendant que de $d$. 
Au terme de cette construction, puisque le degré de $A$ est au plus proportionnel à $c(d) D^{2}$ (comme nous l'avons déjà expliqué plus haut), on obtient la majoration $N \leq c(d) D^{2}$ pour le plus petit degré $N$ d'isogénie liant $E$ et $E^{\sharp}$.

Le polynôme $P$ est construit au moyen du lemme de Thue-Siegel. On a besoin ainsi d'un "processus d'extrapolation" (commun aux démonstrations de transcendance) qui fait appel au lemme de Schwarz ; ceci pour pouvoir accéder à l'inégalité $T \geq c(d) D^{2}$ requise.

On tient compte de la masse de zéros d'une certaine fonction analytique $f$ dans des "grands" disques centrés à l'origine de $\Delta$. Cette fonction analytique est liée plus précisément au polynôme $P$ évalué en des fonctions $\sigma, \wp$ de Weierstrass associées aux réseaux de périodes de $E$ et $E^{\sharp}$.

Comme les fonctions $\sigma$ de Weierstrass ont leur ordre de croissance égal à 2 , il faut que $D \geq c(d) h(E)^{2}$ si $\left|\omega_{2}\right|$ est "beaucoup" plus grand que $\left|\omega_{1}\right|$, ou si $\left|\omega_{2}^{\sharp}\right|$ est "beaucoup" plus grand que $\left|\omega_{1}^{\sharp}\right|$ (cf. lemme 4.3, p. 12 de [Ma-Wü4]). C'est à ce point que l'exposant 4 s'impose dans l'estimation (4).

Justifions maintenant l'exposant 2 de $h(E)$ obtenu dans l'estimation (2) du théorème 1 . Le point essentiel de notre démonstration de transcendance, énoncée précisément dans la proposition 3.1, se situe dans notre lemme de croissance analytique 3.7 , qui améliore de manière décisive le lemme de croissance analytique 4.3, p. 12 de [Ma-Wü4].

Le lemme 3.7 se démontre en utilisant les estimations explicites données par David pour la croissance analytique de fonctions entières associées à la "fonction auxiliaire" sur la droite de Baker.

Pour pouvoir appliquer notre lemme 3.7 nous devons regarder la variété abélienne $G$ comme étant plongée dans un espace multiprojectif $\mathbb{P}=\mathbb{P}_{2}^{4}$ (plongement fixé par les modèles de Weierstrass (1)), et travailler avec des multidegrés et des fonctions de Hilbert-Samuel multihomogènes.

Quitte à choisir de manière "appropriée" $\omega_{1}, \omega_{2}, \omega_{1}^{\sharp}$ et $\omega_{2}^{\sharp}$, si $P$ est un polynôme de multidegré $\left(D_{1}, \ldots, D_{4}\right)$ dans l'anneau de coordonnées multihomogènes de $\mathbb{P}$, alors notre lemme de croissance analytique 3.7 permet de construire le majorant suivant pour la restriction de $|f|$ à la droite de Baker (nous ne retenons que les termes quadratiques en $|z|$ ) :

$$
\exp \left\{c(d)\left(\Im(\tau)^{-1} D_{1}+\Im(\tau) D_{2}+\Im\left(\tau^{\sharp}\right)^{-1} D_{3}+\Im\left(\tau^{\sharp}\right) D_{4}\right)|z|^{2}\right\},
$$

où $\Im(\tau)$ et $\Im\left(\tau^{\sharp}\right)$ sont les parties imaginaires des nombres $\tau=\omega_{2} / \omega_{1}$ et $\tau^{\sharp}=\omega_{2}^{\sharp} / \omega_{1}^{\sharp}$ (cf. paragraphe 2 ci-dessous).

Nous avons pensé à ce point à introduire dans la dépendance des paramètres $\left(D_{j}\right)_{j=1, \ldots, 4}$ les quantités $\Im(\tau)$ et $\Im\left(\tau^{\sharp}\right)$ (cf. choix des paramètres dans la proposition 3.1). Aucun des travaux [Da], [Ma-Wü4] ou [Ma-Wü2] n'utilise ce principe, pourtant assez naturel. 
La dépendance en les quantités $\Im(\tau)$ et $\Im\left(\tau^{\sharp}\right)$ introduit immédiatement des problèmes qui n'apparaissent pas dans les travaux mentionnés ci-dessus.

Contrairement à [Ma-Wü4], nous sommes obligés d'utiliser ce qu'on appelle "l'astuce" de Philippon et Waldschmidt introduite dans [Phi-Wa] $\left({ }^{2}\right)$ (cf. sous-paragraphe 3.1). Elle consiste en ce qui suit.

Nous voulons appliquer le lemme de multiplicités (cf. lemme 4.1, paragraphe 4) pour pouvoir construire une sous-variété abélienne $\mathfrak{H}$ de $G$ satisfaisant une des conditions $(*)$ mentionnées plus haut, pour pouvoir construire ensuite une isogénie de degré bien borné.

Parmi toutes les autres sous-variétés abéliennes il en existe au moins une, $\mathfrak{H}_{0}$, dont les équations permettent de déterminer une majoration pour le rang de la matrice $\mathcal{B}$ dans le lemme de Thue-Siegel. Cette sous-variété abélienne est parfois appelée "la plus mauvaise sous-variété abélienne". Elle est décrite dans le sous-paragraphe 3.1 : plus elle "grande", plus le rang de $\mathcal{B}$ est "petit" (cf. lemme 3.6).

L'astuce de Philippon et Waldschmidt consiste précisément à appliquer cette observation pour pouvoir "éliminer" toutes les sous-variétés abéliennes ne satisfaisant pas les conditions sur les codimensions $(*)$ en demandant un ordre d'annulation $T$ "plus grand", et ceci est possible d'après le contrôle sur le rang de $\mathcal{B}$.

Nous sommes donc ramenés à étudier les sous-variétés abéliennes $\mathfrak{H}$ satisfaisant une des conditions $(*)$. Si $\mathfrak{H}=A$ est la sous-variété abélienne donnée par le lemme de multiplicités, nous avons terminé. Dans les autres deux cas nous rencontrons de nouveaux phénomènes. Quatre sous-variétés abéliennes exceptionnelles apparaissent, et un cas dégénéré doit être traité à part (voir les commentaires après l'hypothèse 3 du sous-paragraphe 2.2). Si $\mathfrak{H}$ est égal à l'une de ces quatre sous-variétés abéliennes, nous ne pouvons pas appliquer le principe de Goursat-Kolchin-Ribet : elles sont étudiées en détail dans le sous-paragraphe 4.1.

Pour traiter ces sous-variétés abéliennes nous avons introduit des arguments probablement liés aux idées employées dans la descente sur la dimension du paragraphe 10, pp. 451-454 de [Ma-Wü2] (cf. lemmes 4.1 et 4.2). Comme $G$ est un produit de courbes elliptiques notre exposition est de plus complètement élémentaire.

Le théorème 2 se déduit essentiellement du théorème 1 . Cette déduction fera l'objet du paragraphe 5. La principale difficulté qui fait que l'on ne peut pas travailler directement avec les hauteurs semi-stables est technique : on ne peut pas imposer les modèles normalisés du lemme 5.3 tout en gardant nos isogénies normalisées (comme tout au long du sous-paragraphe 4.1).

$\left({ }^{2}\right)$ Qui généralise et simplifie les arguments utilisés dans le paragraphe 10, pp. 451-454 dans [Ma-Wü2]. 
En principe cette difficulté pourrait être contournée en travaillant directement avec des modèles entiers, comme le fait Bost dans [Bo]. Ceci permettrait probablement de faire chuter à 2 l'exposant de $d$ dans l'inégalité (3), mais la technique décrite par Bost dans [Bo] (qui suit de plus près l'approche de Masser et Wüstholz dans [Ma-Wü2]) risque de donner des constantes beaucoup moins raisonnables, parce que l'extrapolation est accomplie en plusieurs points de torsion, qui rendent mauvaise la dépendance en le corps de nombres $\left({ }^{3}\right)$. Ceci n'est qu'un problème technique. Cependant, puisque nous suivons de très près le travail de David [Da] (qui est le seul à accomplir la classique extrapolation de transcendance sur les multiplicités d'un seul point de torsion), pour nous les hauteurs naïves sont les hauteurs "naturelles".

Voici l'organisation de ce texte : dans le paragraphe 2 nous rassemblons les outils élémentaires utilisés tout au long de ce texte. Dans le paragraphe 3 est énoncée et démontrée la proposition 3.1, qui correspond au résultat clé de l'application de la méthode de Baker. Le paragraphe 4 est consacré à l'analyse des sous-variétés abéliennes de $G$, et à la déduction du théorème 1 . Dans le paragraphe 5 nous démontrons le théorème 2. Il s'agit essentiellement d'expliciter des comparaisons numériques effectives entre la hauteur de Faltings semi-stable de $E$ et la hauteur naïve $h(E)$.

2. Notations et lemmes préparatoires. Le groupe de Lie des points complexes $E(\mathbb{C})$ est analytiquement isomorphe à $\mathbb{C} / \Lambda$ où $\Lambda$ est le réseau de $\mathbb{C}$ des périodes de la forme différentielle $\frac{d x}{u}$ associée au modèle de Weierstrass (1). Dans la suite nous utiliserons le plongement analytique induit par l'application suivante :

$$
\exp _{E}: \mathbb{C}-\Lambda \rightarrow \mathbb{P}_{2}(\mathbb{C}), \quad z \mapsto\left(\sigma(z)^{3}: \sigma(z)^{3} \wp(z): \sigma(z)^{3} \wp^{\prime}(z)\right),
$$

où l'on a noté $\wp, \sigma$ les fonctions de Weierstrass associées au réseau $\Lambda$ (cf. chapitres 1 et 18 de [Lan]).

Notons $\mathcal{H}$ le demi-plan supérieur $\mathcal{H}=\{z \in \mathbb{C}: \Im(z)>0\}$ et $\mathfrak{F}$ le domaine fondamental usuel pour l'action de $\mathrm{SL}_{2}(\mathbb{Z})$ décrit par exemple dans [Lan]. Dans toute la suite de ce texte nous fixons une base $\left(\omega_{1}, \omega_{2}\right)$ de $\Lambda$ et une base $\left(\omega_{1}^{\sharp}, \omega_{2}^{\sharp}\right)$ de $\Lambda^{\sharp}$ telles que $\tau=\omega_{2} / \omega_{1} \in \mathfrak{F}$ et $\tau^{\sharp}=\omega_{2}^{\sharp} / \omega_{1}^{\sharp} \in \mathfrak{F}$.

Nous travaillons avec des hauteurs, que nous définissons ci-dessous.

Fixons quelques notations supplémentaires. Notons $M_{K}$ un ensemble complet de valeurs absolues non équivalentes sur le corps de nombres $K$ dont les valeurs absolues non archimédiennes $|\cdot|_{v}$ sont ainsi normalisées : $|p|_{v}=1 / p$ si $p$ est un nombre premier tel que $v \mid p$. Nous écrivons $M_{K}^{\infty}$ pour le sous-ensemble de $M_{K}$ constitué des valeurs absolues archimédiennes.

$\left({ }^{3}\right)$ Le même problème se présente quand on veut appliquer la technique des déterminants d'interpolation de Laurent; voir par exemple [Lau]. 


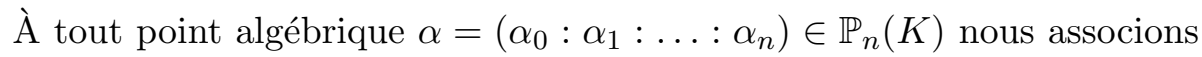
sa hauteur projective logarithmique absolue

$$
h(\alpha)=d^{-1} \sum_{v \in M_{K}} n_{v} \log \max \left\{\left|\alpha_{0}\right|_{v}, \ldots,\left|\alpha_{n}\right|_{v}\right\},
$$

où $n_{v}=\left[K_{v}: \mathbb{Q}_{p}\right]$ est le degré de l'extension $K_{v} \supset \mathbb{Q}_{p}$, où $K_{v}$ et $\mathbb{Q}_{p}$ désignent respectivement les complétés de $K$ en $v$ et de $\mathbb{Q}$ en $p$. Par abus de notation on écrit $h(\alpha)=h(1: \alpha)$ pour $\alpha \in K$; nous renvoyons à [Wa2], chapitre III, pour les propriétés principales. Pour tout polynôme $P$ à coefficients algébriques, sa hauteur $h(P)$ sera par définition la hauteur du point projectif dont les coordonnées sont 1 et les coefficients de $P$.

Dans tout ce texte nous allons en particulier appliquer systématiquement une inégalité élémentaire, souvent sans en faire mention :

InÉgalité De Liouville. Pour tout nombre algébrique non nul $\alpha$, et pour toute place $v$ de $\mathbb{Q}(\alpha)$,

$$
\log |\alpha|_{v} \geq-[\mathbb{Q}(\alpha): \mathbb{Q}] h(\alpha) .
$$

2.1. Inégalités élémentaires. Notons $y=\Im(\tau)$.

Lemme 2.1. Supposons E sans multiplication complexes. On a les estimations suivantes :

(i) $y \leq \frac{3}{2} \max \left\{1, d h\left(j_{E}\right)\right\}$,

(ii) $\exp \{1-d h(E)\} \leq\left|\omega_{1}\right| \leq \exp \left\{1+\frac{1}{4} d h(E)\right\}$,

(iii) $\left|\omega_{2}\right| \leq \exp \left\{\frac{3}{2}+\frac{2}{3} d h(E)\right\}$.

Démonstration. (i) Cette inégalité se démontre en utilisant l'inégalité

$$
y \leq \frac{3}{2} \log \max \{e,|j(\tau)|\},
$$

issue du lemme 1(iv) de [Fa-Ph], et en appliquant l'inégalité triviale $\log \left|j_{E}\right|$ $\leq d h\left(j_{E}\right)$.

(ii) Majoration. Nous avons supposé $E$ sans multiplication complexe, nous avons donc $g_{2} \neq 0$. Le lemme 3.2, p. 27 de [Da] implique, en appliquant l'inégalité de Liouville (5) et la minoration $y \geq \sqrt{3} / 2$ (valable pour tout $\tau=x+i y \in \mathfrak{F})$,

$$
\begin{aligned}
\left|\omega_{1}\right| & \leq \exp \left\{1.85-\frac{1}{6} \pi y-\frac{1}{4} \log (12)+\frac{1}{12} \log |j(\tau)|-\frac{1}{4} \log \left|g_{2}\right|\right\} \\
& \leq \exp \left\{0.78+\frac{d}{4}\left(h\left(g_{2}\right)+h\left(j_{E}\right)\right)\right\} .
\end{aligned}
$$

Minoration. On utilise encore une fois le lemme 3.2 de [Da] :

$$
\left|\omega_{1}\right| \geq \exp \left\{1.825-\frac{1}{6} \pi y-\frac{1}{4} \log (12)+\frac{1}{12} \log |j(\tau)|-\frac{1}{4} \log \left|g_{2}\right|\right\}
$$




$$
\geq \exp \left\{1.20-d \max \left\{1, h\left(j_{E}\right)\right\}\left(\frac{\pi}{4}+\frac{1}{12}\right)-\frac{d}{4} h\left(g_{2}\right)\right\},
$$

en utilisant une fois de plus l'inégalité de Liouville (5).

(iii) On a $\omega_{2}=\tau \omega_{1}$. Comme $|\tau| \leq(\sqrt{10} / 2) d h(E)$, en utilisant les points (i) et (ii), on vérifie bien cette inégalité, ce qui achève la preuve du lemme 2.1.

2.2. Isogénies. Une $K$-isogénie de $E$ vers $E^{\sharp}$ (ou isogénie définie sur $K$ ) est une isogénie $E \rightarrow E^{\sharp}$, stable par le groupe de Galois $\operatorname{Gal}(\bar{K} \mid K)$.

Remarque fondamentale. Supposons $E$ sans multiplication complexe. Dans tout ce texte nous utiliserons très souvent le fait suivant. Toute isogénie comme dans les énoncés des théorèmes 1 et 2 est en fait définie sur $K$. Pour le voir on pourra suivre les arguments de [Ma-Wü4], lemme 6.1, p. 18.

DÉfinition. On dit qu'une isogénie liant deux courbes elliptiques est cyclique si son noyau est un groupe cyclique.

Soient $\left.E\right|_{K}$ et $\left.E^{\sharp}\right|_{K}$ deux courbes elliptiques isogènes et sans multiplication complexe; l'ensemble $\operatorname{Hom}_{K}\left(E^{\sharp}, E\right)$ dont les éléments sont l'application constante $E^{\sharp} \rightarrow 0$ (0 est l'origine de $E$ ) et les isogénies définies sur $K$, a une structure de $\mathbb{Z}$-module libre de rang 1 .

L'image de l'application degré $\operatorname{deg}: \operatorname{Hom}_{K}\left(E^{\sharp}, E\right) \rightarrow \mathbb{Z}$ est de la forme $\left\{N n^{2}: n \in \mathbb{Z}\right\}$ où $N>0$ est le degré minimal d'isogénie. Au plus deux isogénies dans $\operatorname{Hom}_{K}\left(E^{\sharp}, E\right)$ ont leur degré égal à $N$.

En utilisant le lemme 6.2, p. 18 de [Ma-Wü4] on vérifie que, pour qu'une isogénie $\phi: E^{\sharp} \rightarrow E$ soit cyclique, il faut et il suffit qu'elle ait son degré minimal.

Soient $E$ et $E^{\sharp}$ deux courbes elliptiques de réseaux $\Lambda=\omega_{1} \mathbb{Z}+\omega_{2} \mathbb{Z}$ et $\Lambda^{\sharp}=\omega_{1}^{\sharp} \mathbb{Z}+\omega_{2}^{\sharp} \mathbb{Z}$ respectivement, avec $\tau=\omega_{2} / \omega_{1} \in \mathfrak{F}$ et $\tau^{\sharp}=\omega_{2}^{\sharp} / \omega_{1}^{\sharp} \in \mathfrak{F}$. Supposons-les isogènes. Soit $\psi: E^{\sharp} \rightarrow E$ une isogénie. Il existe un unique nombre complexe non nul $\alpha$ tel que $\alpha \Lambda^{\sharp} \subset \Lambda$ et tel que le diagramme suivant soit commutatif (les flèches verticales sont les projections usuelles $\mathbb{C} \rightarrow \mathbb{C} / \Lambda^{\sharp}$ et $\mathbb{C} \rightarrow \mathbb{C} / \Lambda$ respectivement $)$ :

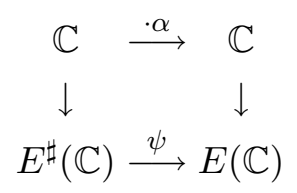

DÉfinition. Si $\alpha=1$ nous dirons que l'isogénie $\psi$ est normalisée.

On vérifie dans ce cas qu'il existe une unique matrice $\left(m_{i, j}\right)_{1 \leq i, j \leq 2} \in$ $\mathrm{GL}_{2}(\mathbb{C})$ à coefficients entiers rationnels (de déterminant égal au degré de 
$\psi)$, donnant lieu à deux relations :

$$
\begin{aligned}
& \omega_{1}^{\sharp}=m_{1,1} \omega_{1}+m_{1,2} \omega_{2}, \\
& \omega_{2}^{\sharp}=m_{2,1} \omega_{1}+m_{2,2} \omega_{2} .
\end{aligned}
$$

HYPOTHÈSE 1. Dans toute la suite de ce texte nous supposerons que les courbes elliptiques $E$ et $E^{\sharp}$ sont sans multiplication complexe.

Cette hypothèse ne limite pas la généralité de notre résultat. Dans [Ma-Wü4], pp. 21-22, on pourra trouver une esquisse de démonstration d'un résultat aussi précis que notre théorème 1 en la dépendance en la hauteur, mais valable uniquement pour des courbes elliptiques à multiplication complexe. Nous avons donné une preuve détaillée et numériquement explicite dans $[\mathrm{Pe}]\left({ }^{4}\right)$.

Rappelons seulement que pour traiter le cas de courbes elliptiques à multiplication complexe, on peut appliquer les idées classiques de D. V. et G. V. Chudnovsky comme dans [Chu] et travailler dans $G^{\prime}=E \times E^{\sharp}$ où, comme nous l'avons déjà remarqué, l'image de la droite de Baker est une courbe elliptique.

НyPothèse 2. Il existe une isogénie normalisée et cyclique $\psi: E^{\sharp} \rightarrow E$.

Nous lèverons cette hypothèse dans le sous-paragraphe 4.2 .

Hypothèse 3. L'isogénie normalisée $\psi: E^{\sharp} \rightarrow E$ est telle que $m_{1,2} \neq 0$.

Nous appelons le cas où $m_{1,2}=0$, cas dégénéré. Notre démonstration utilisant la variété abélienne $G=E^{2} \times E^{\sharp 2}$ ne fonctionne pas si $m_{1,2}=0$ (voir par exemple le lemme 4.1).

Malgré ce fait, ce cas ne pose aucune difficulté : Il s'agit de reprendre toute la démonstration en travaillant avec la seule relation linéaire $\omega_{1}^{\sharp}=$ $m_{1,1} \omega_{1}$. On se place alors dans la variété abélienne $G^{\prime}=E \times E^{\sharp}$ : cela peut se faire par des arguments classiques, qui s'appliquent aussi au cas de la multiplication complexe. Aussi il résulte que les constantes dans les estimations sont bien meilleures. Nous ne traiterons donc pas ce cas dans ce texte : pour cela voir le paragraphe 5 de [Pe] ou pp. 21-22 de [Ma-Wü4], ou encore [Chu]. Les deux hypothèses 1 et 3 sont donc de natures similaires.

\subsection{Lemme d'isogénie : principe de Goursat-Kolchin-Ribet}

DÉfinition. Fixons deux entiers positifs $n_{1}, n_{2} \geq 1$ et soit $\mathfrak{H}$ une sousvariété abélienne de $E^{n_{1}} \times E^{\sharp n_{2}}$. On dit que $\mathfrak{H}$ est non déployée si elle ne

$\left({ }^{4}\right)$ Pour deux courbes elliptiques $E$ et $E^{\sharp}$ définies sur un corps de nombres $K$, isogènes sur $\bar{K}$ avec $\operatorname{End}_{\bar{K}}(E) \neq \mathbb{Z}$, on devrait pouvoir construire deux $\bar{K}$-isogénies de $E^{\sharp}$ vers $E$ qui sont $\mathbb{Z}$-linéairement indépendantes et dont le produit des degrés soit majoré par $h(E)^{4}$ à une constante explicite près ne dépendant que de $[K: \mathbb{Q}]$. 
s'écrit pas comme produit $\mathfrak{H}_{1} \times \mathfrak{H}_{2}$ où $\mathfrak{H}_{1}$ est une sous-variété abélienne de $E^{n_{1}}$ et $\mathfrak{H}_{2}$ une sous-variété abélienne de $E^{\sharp n_{2}}$.

Le lemme suivant est une version légèrement modifiée du lemme d'isogénie de [Ma-Wü4] (cas $\left.n_{1}=n_{2}=1\right)$.

LEMME 2.2 (Lemme d'isogénie). Soient $E_{1}$ et $E_{2}$ deux courbes elliptiques et soit $\mathfrak{H}$ une sous-variété abélienne de $E_{1} \times E_{2}$ non déployée. Les sous-groupes $\mathfrak{H}_{1}=\mathfrak{H} \cap\left(E_{1} \times 0_{2}\right)$ et $\mathfrak{H}_{2}=\mathfrak{H} \cap\left(0_{1} \times E_{2}\right)$ sont alors finis $;$ notons $h_{1}$ et $h_{2}$ leurs cardinaux respectifs. Il existe alors une isogénie $\psi: E_{1} \rightarrow E_{2}$ de degré $\leq h_{1} h_{2}$.

Démonstration. Notons $\pi_{1}: E_{1} \times E_{2} \rightarrow E_{1}$ et $\pi_{2}: E_{1} \times E_{2} \rightarrow E_{2}$ les projections $\pi_{1}\left(x_{1}, x_{2}\right)=x_{1}$ et $\pi_{2}\left(x_{1}, x_{2}\right)=x_{2}$. Comme $\mathfrak{H}$ est non déployée la projection $\pi_{1}$ induit une isogénie $\pi_{1}: \mathfrak{H} \rightarrow E_{1}$ dont le noyau est $\mathfrak{H}_{2}$. De même la projection $\pi_{2}$ induit une isogénie $\pi_{2}: \mathfrak{H} \rightarrow E_{2}$ dont le noyau est $\mathfrak{H}_{1}$.

L'isogénie $\psi: E_{1} \rightarrow E_{2}$ définie par $\psi=\pi_{2} \circ \widehat{\pi}_{1}$ (composée de la duale de $\pi_{1}$ avec $\pi_{2}$ ) a son degré égal à $h_{1} h_{2}$ et le lemme est démontré.

\section{Partie arithmético-analytique : application de la méthode de}

Baker. Dans ce paragraphe nous démontrons le résultat de transcendance (proposition 3.1) ci-dessous.

Nous allons fixer d'abord un certain nombre de conventions et introduire des objets que l'on utilisera dans la suite.

Nous utilisons des multi-indices dans $\mathbb{N}^{4}$ que nous soulignerons. Exemple : $\underline{D}=\left(D_{1}, D_{2}, D_{3}, D_{4}\right)$. Nous définissons une relation d'ordre entre multi-indices : $\underline{\lambda} \leq \underline{D}$ si et seulement si pour tout $j=1, \ldots, 4$ on a $\lambda_{j} \leq D_{j}$. Parfois nous adopterons les mêmes conventions pour des paquets d'indéterminées comme $\underline{X}=\left(X_{1}, X_{2}, X_{3}, X_{4}\right), K[\underline{X}]:=K\left[X_{1}, X_{2}, X_{3}, X_{4}\right]$. Le symbole $\underline{X}^{\underline{\lambda}}$ aura la signification suivante $: \underline{X} \underline{\underline{\lambda}}:=X_{1}^{\lambda_{1}} X_{2}^{\lambda_{2}} X_{3}^{\lambda_{3}} X_{4}^{\lambda_{4}}$.

On notera en outre $\mathcal{D}_{\underline{D}}$ l'ensemble des monômes unitaires $\underline{X} \underline{\lambda}$ de multidegré $\underline{\lambda} \leq \underline{D}$; son cardinal est égal à $\prod_{n}\left(D_{n}+1\right)$.

Soit $\Lambda^{\sharp}$ le réseaux des périodes de $E^{\sharp}$. Fixons une base $\left(\omega_{1}^{\sharp}, \omega_{2}^{\sharp}\right)$ de $\Lambda^{\sharp}$ telle que $\tau^{\sharp}=\omega_{1}^{\sharp} / \omega_{2}^{\sharp} \in \mathfrak{F}$. Notons $y^{\sharp}=\Im\left(\tau^{\sharp}\right)$. Nous utiliserons aussi le vecteur $\left(\zeta_{1}, \zeta_{2}, \zeta_{3}, \zeta_{4}\right)=\left(y^{-1}, y, y^{\sharp-1}, y^{\sharp}\right)$.

Posons

$$
\mathcal{K}=\mathbb{Q}\left(g_{2}, g_{2}^{\sharp}, \wp\left(\omega_{1} / 2\right), \wp\left(\omega_{2} / 2\right), \wp^{\sharp}\left(\omega_{1}^{\sharp} / 2\right), \wp^{\sharp}\left(\omega_{2}^{\sharp} / 2\right)\right) .
$$

Grâce à l'identité $\wp^{\prime}\left(\omega_{1} / 2\right)=\wp^{\prime}\left(\omega_{2} / 2\right)=\wp^{\prime}\left(\left(\omega_{1}+\omega_{2}\right) / 2\right)=0$ nous savons que les coordonnées projectives de tous les points de 2-torsion de $E$ et $E^{\sharp}$ sont définies sur $\mathcal{K}$. De plus, $g_{3}, g_{3}^{\sharp} \in \mathcal{K}$, ce qui implique $K \supset \mathcal{K}$.

Nous faisons, en plus des hypothèses 1,2 et 3 , une hypothèse supplémentaire qui est naturelle pour la démonstration de la proposition 3.1.

Hypothèse 4. Nous supposons que $K=\mathcal{K}$. 
Nous lèverons cette hypothèse dans le sous-paragraphe 4.2.

Notons $h=h(E)+h\left(E^{\sharp}\right)$ et $\mathcal{L}=h+\log N$. Notons [·] la partie entière sur $\mathbb{R}$, et $e=2.71 \ldots$ le nombre d'Euler.

Soient $z_{1}, z_{2}, z_{1}^{\sharp}$ et $z_{2}^{\sharp}$ quatre variables complexes, notons $Z$ le sous-espace vectoriel de $T_{\mathbb{C}} \cong \mathbb{C}^{4}$ de dimension 2 sur $\mathbb{C}$ qui est l'intersection des deux hyperplans $Z_{1}, Z_{2}$ définis par les équations linéaires :

$$
Z_{1}: z_{1}^{\sharp}=m_{1,1} z_{1}+m_{1,2} z_{2}, \quad Z_{2}: z_{2}^{\sharp}=m_{2,1} z_{1}+m_{2,2} z_{2}
$$

et notons

$$
\partial_{1}=\omega_{1} \frac{\partial}{\partial z_{1}}+\omega_{2} \frac{\partial}{\partial z_{2}}, \quad \partial_{2}=\omega_{2} \frac{\partial}{\partial z_{1}}-\omega_{1} \frac{\partial}{\partial z_{2}} .
$$

Soient $t, t_{0}$ deux entiers positifs. Notons $\mathcal{T}_{t_{0}, t}$ l'ensemble des monômes différentiels $\partial_{1}^{\mu_{1}} \partial_{2}^{\mu_{2}}$ avec $0 \leq \mu_{1}+\mu_{2} \leq t, 0 \leq \mu_{2} \leq t_{0}$. Lorsque $t_{0} \geq t$ on écrira simplement $\mathcal{T}_{t}$. L'ensemble $\mathcal{T}_{t_{0}, t}$ a un cardinal

$$
(1 / 2)\left(2 t+2-\min \left\{t, t_{0}\right\}\right)\left(\min \left\{t, t_{0}\right\}+1\right)>0 .
$$

Proposition 3.1. Supposons que les hypothèses du théorème 1 et les hypothèses 1, 2, 3 et 4 soient satisfaites. On peut trouver un nombre réel $\mathcal{V}$ satisfaisant l'encadrement suivant:

$$
1 \leq \mathcal{V} \leq 285 d^{2} \mathcal{L}^{2}
$$

tel que si l'on définit cinq entiers positifs de la manière suivante:

$$
\begin{gathered}
D_{n}=\left[10^{18} d \log \max \{e, d\} \mathcal{L} \zeta_{n}^{-1}\right] \quad \text { pour } n=1, \ldots, 4, \\
T=\left[2.46 \cdot 10^{38} \mathcal{V} d^{2}(\log \max \{e, d\})^{2} \mathcal{L}^{2}\right],
\end{gathered}
$$

alors il existe un polynôme non nul $P \in K[\underline{X}]$ de multidegré $\leq \underline{D}=$ $\left(D_{1}, D_{2}, D_{3}, D_{4}\right)$ tel que la fonction abélienne

$$
f_{P}\left(z_{1}, z_{2}\right)=P\left(\wp\left(z_{1}\right), \wp\left(z_{2}\right), \wp^{\sharp}\left(m_{1,1} z_{1}+m_{1,2} z_{2}\right), \wp^{\sharp}\left(m_{2,1} z_{1}+m_{2,2} z_{2}\right)\right)
$$

satisfasse $\partial f_{P}\left(\omega_{1} / 2, \omega_{2} / 2\right)=0$ pour tout $\partial \in \mathcal{T}_{T}$.

Avec $T$ et $\left(D_{j}\right)_{j=1, \ldots, 4}$ il y a encore quatre paramètres qui interviendront dans la démonstration de la proposition 3.1 ; nous regroupons tous les paramètres ci-dessous et une fois pour toutes.

Posons $C_{1}=10^{18}, C_{2}=2.46 \cdot 10^{38}$ et

$$
\begin{aligned}
& C_{3}=41.66 C_{2} C_{1}^{-1 / 2}\left(\log C_{1}\right)^{1 / 2}, \\
& C_{4}=0.48 C_{1}^{1 / 2}\left(\log C_{1}\right)^{1 / 2}, \\
& C_{5}=72 C_{2} \log C_{1} \\
& C_{6}=1.53 C_{2} \log C_{1} .
\end{aligned}
$$


Les paramètres sont :

$$
\begin{aligned}
D_{n} & =\left[C_{1} d \log \max \{e, d\} \mathcal{L} \zeta_{n}^{-1}\right], \quad n=1, \ldots, 4, \\
T & =\left[C_{2} \mathcal{V} d^{2}(\log \max \{e, d\})^{2} \mathcal{L}^{2}\right], \\
T_{0} & =\left[C_{3} \mathcal{V} d^{2}(\log \max \{e, d\})^{2} \mathcal{L}^{2}\right], \\
S & =\left[C_{4} d \log \max \{e, d\} \mathcal{L}\right], \\
U & =C_{5} \mathcal{V} d^{3}(\log \max \{e, d\})^{3} \mathcal{L}^{3}, \\
Q & =C_{6} \mathcal{V} d^{2}(\log \max \{e, d\})^{3} \mathcal{L}^{3} .
\end{aligned}
$$

La construction du polynôme $P$ de la proposition 3.1 sera accomplie en deux étapes.

Dans la première étape (sous-paragraphe 3.2) nous montrons qu'il est possible de construire un polynôme $P \in \mathbb{C}[\underline{X}]$ de multidegré $\leq \underline{D}$ et dont les valeurs absolues des coefficients sont $\leq e^{d+2 d^{2} h+Q}\left({ }^{5}\right)$, satisfaisant

$$
\left|\partial f_{P}\left(\omega_{1} / 2, \omega_{2} / 2\right)\right| \leq e^{-U}
$$

pour tout $\partial \in \mathcal{T}_{T_{0}, 2 T}$.

C'est le principe des tiroirs, sous la forme d'un lemme de Thue-Siegel, qui nous fournit ce polynôme. Pour les raisons esquissées dans le sousparagraphe 1.2, un exposant de Dirichlet non égal à 1 apparaît comme indispensable si l'on veut traiter la partie algébrique qui suit.

Afin de procéder à la construction de $P$ et de prouver donc le lemme 3.2, il faut déterminer la quantité $\mathcal{V}$ de la proposition 3.1 (ce sera fait dans le lemme 3.1), puis estimer le rang du système linéaire associé à des conditions caractérisant $P$ (lemme 3.6).

Ces deux calculs sont effectués en fonction d'une "plus mauvaise" sousvariété abélienne de $G$, dont la définition est naturelle à partir du lemme de multiplicités (le lemme 4.1 du paragraphe suivant), et dont les propriétés principales sont étudiées dans le sous-paragraphe 3.1 ci-dessous.

Dans la deuxième étape (sous-paragraphe 3.3 ) on applique essentiellement l'inégalité de Liouville et le lemme de Schwarz en une variable, mais comme la multiplicité en $\left(\omega_{1} / 2, \omega_{2} / 2\right)$ est assez faible, nous devons exploiter la périodicité de la fonction $f_{P}\left(\omega_{1} z, \omega_{2} z\right)$ (comme le font déjà Masser et Wüstholz dans [Ma-Wü4]). Ceci nous mène à étudier la croissance analytique d'une fonction entière $\left(\Theta \cdot f_{P}\right)\left(\omega_{1} z, \omega_{2} z\right)$ sur un disque de rayon $S$ centré en $\left(\omega_{1} / 2, \omega_{2} / 2\right)$, et à établir un lemme de croissance analytique (le lemme 3.7) suffisamment précis pour la situation. C'est ici aussi que l'on exploite la flexibilité de l'usage des multidegrés $\underline{D}$.

Du côté des estimations arithmétiques nous procédons de manière totalement standard. Nous préférons toutefois détailler toutes les démonstrations. bas.

$\left(^{5}\right)$ Cette inégalité provient du choix d'une base de $K$ sur $\mathbb{Q}$ que nous allons fixer plus 
3.1. Prélude : la plus mauvaise sous-variété abélienne. Étant fixé un espace multiprojectif complexe $\mathbb{P}(\mathbb{C})=\left(\mathbb{P}_{N_{1}} \times \ldots \times \mathbb{P}_{N_{p}}\right)(\mathbb{C})$ et une sousvariété $V \subset \mathbb{P}(\mathbb{C})$ de dimension $b$, nous adoptons la définition de degré partiel $\operatorname{deg}_{\underline{\alpha}}(V)$ relatif à un multi-indice $\underline{\alpha} \leq \underline{N}$ tel que $|\underline{\alpha}|=\sum_{n=1}^{p} \alpha_{n}=b$ donnée dans [Phi2], p. 362. Pour des réels positifs $D_{1}, \ldots, D_{p}$ on notera $\mathcal{H}\left(V: D_{1}, \ldots, D_{p}\right)$ la partie homogène de plus haut degré du polynôme de Hilbert-Samuel multi-homogène, multipliée par $b$ !. On a l'identité

$$
\mathcal{H}\left(V: D_{1}, \ldots, D_{p}\right)=\sum_{|\underline{\alpha}|=b} \operatorname{deg}_{\underline{\alpha}}(V) \frac{b !}{\alpha_{1} ! \ldots \alpha_{p} !} D_{1}^{\alpha_{1}} \ldots D_{p}^{\alpha_{p}} .
$$

Dans le cas de $G=E \times E \times E^{\sharp} \times E^{\sharp}$ plongée de la manière usuelle dans $\mathbb{P}_{2}(\mathbb{C})^{4}$, notons $A=\exp _{G}(Z)$ (nous rappelons que $Z$ a été défini dans (8)) et soit $\Delta$ le sous-espace vectoriel de $T_{\mathbb{C}} \cong \mathbb{C}^{4}$ engendré par $\left(\omega_{1}, \omega_{2}, \omega_{1}^{\sharp}, \omega_{2}^{\sharp}\right)$. Nous sommes dans le cas sans multiplication complexe et on montre sans difficulté que l'adhérence de Zariski de $\exp _{G}(\Delta)$ est exactement $A$. Nous allons nous intéresser aux sous-variétés abéliennes de $G$. Pour $\mathfrak{H}$ une telle sous-variété abélienne, et pour un multi-indice $\underline{D}=\left(D_{1}, D_{2}, D_{3}, D_{4}\right)$, nous étudions $\mathcal{H}(\mathfrak{H}: \underline{D})$.

Soit $\mathfrak{A}$ l'ensemble de toutes les sous-variétés abéliennes $\mathfrak{H} \subset G$ satisfaisant une des propriétés suivantes : ou bien $\mathfrak{H} \subset A$ et $\operatorname{codim}_{A}(\mathfrak{H})=1$, ou bien $A \subset \mathfrak{H}$ et $\operatorname{codim}_{\mathfrak{H}}(A)=1$, ou bien $\mathfrak{H}=A$.

LEMME 3.1. Il existe un nombre rationnel $0<\varepsilon<10^{-5}$ et un nombre réel $\mathcal{V}$ satisfaisant l'encadrement

$$
1 \leq \mathcal{V} \leq 285 d^{2} \mathcal{L}^{2}
$$

tels que l'on ait, avec le choix des paramètres (10):

(i) Pour toute sous-variété abélienne $\mathfrak{H} \subset G$ telle que $\mathfrak{H} \notin \mathfrak{A}$,

$$
\left(\begin{array}{c}
{[(T-1) / 4]+\varrho} \\
\varrho
\end{array}\right) \mathcal{H}(\mathfrak{H}: \underline{D}) \geq(1+\varepsilon) \mathcal{H}(G: \underline{D})
$$

où l'on a posé $\varrho=\operatorname{codim}_{A}(\mathfrak{H} \cap A)$.

(ii) Il existe une sous-variété abélienne $\mathfrak{H}_{0} \subset G$ telle que $\mathfrak{H}_{0} \notin \mathfrak{A}$ et vérifiant

$$
\left(\begin{array}{c}
{[(T-1) / 4]+\varrho_{0}} \\
\varrho_{0}
\end{array}\right) \mathcal{H}\left(\mathfrak{H}_{0}: \underline{D}\right)=(1+\varepsilon) \mathcal{H}(G: \underline{D}),
$$

où l'on a posé $\varrho_{0}=\operatorname{codim}_{A}\left(\mathfrak{H}_{0} \cap A\right)$.

Si $\mathfrak{H}_{0} \neq 0$ alors $\varrho_{0}=1$ et $\operatorname{dim} \mathfrak{H}_{0}=2$.

Démonstration. Considérons le choix des paramètres (10) et remplaçons temporairement $\mathcal{V}$ par une variable réelle positive $\mathcal{V}^{\prime}$. Notons que la quantité 
$T$ dépend de $\mathcal{V}^{\prime}$, tandis que le quadruplet $\underline{D}=\left(D_{1}, \ldots, D_{4}\right)$ n'en dépend pas.

Nous choisissons $\mathfrak{H}_{0}$ comme étant une des sous-variétés abéliennes n'appartenant pas à $\mathfrak{A}$ et telle que la quantité $\mathcal{H}\left(\mathfrak{H}_{0}: \underline{D}\right)$ soit la plus petite possible.

En faisant varier $\mathcal{V}^{\prime}$ dans le choix du paramètre $T$ effectué dans (10) nous trouvons une unique valeur $\mathcal{V}^{\prime}=\mathcal{V}$ telle que l'égalité (14) soit vérifiée pour un nombre rationnel $0<\varepsilon<10^{-5}$.

Nous voyons donc que les deux conditions (i) et (ii) sont satisfaites et il ne nous reste qu'à montrer que la quantité $\mathcal{V}$ ainsi trouvée satisfait l'encadrement (12).

Tout d'abord, pour $\mathfrak{H}=0$ et d'après (i) on a

$$
\left(\begin{array}{c}
{[(T-1) / 4]+2} \\
2
\end{array}\right) \geq \mathcal{H}(G: \underline{D})=4 ! \cdot 3^{4} \prod_{n} D_{n} .
$$

On en tire une minoration pour $\mathcal{V}$ que l'on vérifie aisément être exactement la minoration dans (12), en utilisant le choix des paramètres (10).

Démontrons la majoration dans (12). En travaillant directement avec l'identité (14) et en raisonnant en particulier sur la dimension de $\mathfrak{H}_{0}$ ainsi que sur sa codimension $\varrho_{0}$ dans $A$, nous vérifions que l'on peut se restreindre aux cas suivants : $\mathfrak{H}_{0}=0$ et $\varrho_{0}=1$; dans cette dernière situation on vérifie aussi que $\mathfrak{H}_{0}$ a pour dimension 2 et par des raisons d'adhérence, $\mathfrak{H}_{0} \not \supset \exp _{G}(\Delta)$ (pas de multiplication complexe).

Si $\mathfrak{H}_{0}=0$ alors $\mathcal{V}=1$. Nous devons nous soucier uniquement du deuxième cas. D'après l'identité (14) on a

$$
(1+\varepsilon) 4 ! \cdot 3^{4} \prod_{n} D_{n} \geq \min \left\{D_{1} D_{3}, D_{2} D_{4}\right\}(T / 4) .
$$

Pour prouver cette inégalité il suffit d'observer que tous les autres monômes unitaires de degré 2 en les $D_{j}$ prennent des valeurs certainement plus petites que $\min \left\{D_{1} D_{3}, D_{2} D_{4}\right\}$, à cause de la dépendance en $y$ et $y^{\sharp}$ dans le choix des paramètres (10). Comme

$$
\min \left\{D_{1} D_{3}, D_{2} D_{4}\right\} \geq 10^{36} d^{2}(\log \max \{e, d\})^{2} \mathcal{L}^{2} \min \{1, y\}^{-1} \min \left\{1, y^{\sharp}\right\}^{-1},
$$

on parvient à l'estimation suivante en appliquant l'inégalité (i) du lemme 2.1 :

$$
\mathcal{V} \leq 69984(1+\varepsilon) C_{1}^{2} C_{2}^{-1} d^{2} \mathcal{L}^{2},
$$

d'où le lemme 3.1 se déduit aisément.

Remarque. On peut certainement raffiner cette estimation. Cependant nous n'aurons besoin dans la suite du texte que de majorations pour $\log \mathcal{V}$. On peut montrer dans le lemme 3.1 que si $\mathfrak{H}_{0} \neq 0$ alors on a seulement deux 
possibilités en supposant l'hypothèse 3 satisfaite :

$$
\mathfrak{H}_{0}=0 \times E \times H_{2}, \quad \mathfrak{H}_{0}=H_{1} \times 0^{\sharp} \times E^{\sharp},
$$

où $H_{1}=\exp _{E^{\sharp} \times E^{\sharp}}\left(\widetilde{Z}_{1}\right)$ et $H_{2}=\exp _{E \times E}\left(\widetilde{Z}_{2}\right)$, avec $\widetilde{Z}_{1}$ (resp. $\left.\widetilde{Z}_{2}\right)$ lieu d'annullation de la forme $m_{2,2} z_{1}^{\sharp}-m_{1,2} z_{2}^{\sharp}$ (resp. $m_{1,1} z_{1}+m_{1,2} z_{2}$ ). Nous n'utiliserons pas ces détails, mais remarquons à partir de cela que si on avait des inégalités de type $y \leq c_{4}\left(y^{\sharp}+N^{1-\varepsilon}\right)$ et $y^{\sharp} \leq c_{4}\left(y+N^{1-\varepsilon}\right)$ pour une place de $K$ et pour une constante numérique $c_{4}$ ne dépendant que de $K$ ( $\varepsilon$ est cette fois un réel positif), alors on pourrait éviter l'application de l'astuce de Philippon et Waldschmidt (quitte éventuellement à accepter une dépendance plus mauvaise en $d$ ). On serait alors essentiellement ramenés au cas où $\mathfrak{H}_{0}=0$.

Malheureusement les inégalités sur $y$ et $y^{\sharp}$ ci-dessus ne semblent pas être valables en général. Par contre la hauteur de Faltings $h(E)$ - dont les quantités $y^{\sigma}$ associées aux plongements $\sigma$ de $K$ dans $\mathbb{C}$ correspondent à des contributions locales à l'infini — satisfait $h(E) \leq h\left(E^{\sharp}\right)+(1 / 2) \log N$.

Le lecteur se convaincra que l'utilisation de l'astuce de Philippon et Waldschmidt est indispensable.

\subsection{Premier pas : Lemme de Thue-Siegel}

Lemme 3.2. Il existe un polynôme non nul $P \in K[\underline{X}]$ de multidegré $\leq \underline{D}$ dont le maximum des valeurs absolues des coefficients est majoré par $e^{d+2} d^{2} h+Q$ et de hauteur satisfaisant $h(P) \leq 1.5 d^{2} h+8 d^{2} \log 2+Q+\log d$, tel que pour tout monôme différentiel $\partial=\partial_{1}^{\mu_{1}} \partial_{2}^{\mu_{2}}$ avec $0 \leq \mu_{1}+\mu_{2} \leq 2 T$ et $0 \leq \mu_{1} \leq T_{0}$ on ait

$$
\left|\partial f_{P}\left(\omega_{1} / 2, \omega_{2} / 2\right)\right| \leq e^{-U} .
$$

Nous pouvons voir notre problème comme la résolution d'un système linéaire en $L$ inconnues et $\widetilde{T}$ inéquations linéaires où $L$ est le nombre de monômes dans l'écriture de $P$ multiplié par $d$, et

$$
T=\operatorname{Card} \mathcal{T}_{T_{0}, 2 T}=(1 / 2)\left(T_{0}+1\right)\left(2+2 T-T_{0}\right) .
$$

Nous voulons trouver une solution entière rationnelle de ce système linéaire, de hauteur logarithmique plus petite que $Q$. Nous appliquons le lemme 6.1, p. 301 de [Phi-Wa] (Lemme de Thue-Siegel "approché"), que nous reproduisons en adaptant les notations.

Lemme 3.3. Soit $\mathcal{B} \in \operatorname{Mat}_{L, \widetilde{T}}(\mathbb{C})$ une matrice de nombres complexes à $L$ lignes et $\widetilde{T}$ colonnes, de rang $\leq \varsigma$. Soient $Q, U, M \geq 0$ des nombres réels tels que

$$
\left[2 \widetilde{T} e^{Q+M+U}+1\right]^{2 \varsigma} \leq e^{Q L}
$$

et

$$
\max _{1 \leq j \leq \widetilde{T}}\left\{\sum_{i=1}^{L}\left|\mathcal{B}_{i, j}\right|\right\} \leq e^{M} .
$$


Il existe un vecteur non nul $\left(p_{1}, \ldots, p_{L}\right)$ de $\mathbb{Z}^{L}$ tel que $\max _{1 \leq i \leq L}\left\{\left|p_{i}\right|\right\} \leq e^{Q}$ et

$$
\max _{1 \leq j \leq \widetilde{T}}\left\{\left|\sum_{i=1}^{L} \mathcal{B}_{i, j} p_{i}\right|\right\} \leq e^{-U}
$$

Choix d'une base pour $K$. Suivant le paragraphe 6.6, p. 71 de [Da], nous choisissons une fois pour toutes une base $\mathcal{A}=\left\{a_{1}, \ldots, a_{d}\right\}$ de $K$ sur $\mathbb{Q}$ constituée de monômes unitaires en les quantités

$$
g_{2}, g_{2}^{\sharp}, \wp\left(\omega_{1} / 2\right), \wp\left(\omega_{2} / 2\right), \wp^{\sharp}\left(\omega_{1}^{\sharp} / 2\right), \wp^{\sharp}\left(\omega_{2}^{\sharp} / 2\right),
$$

telle que la hauteur projective du point $\left(1: a_{1}: \ldots: a_{d}\right)$ soit majorée par :

$$
h\left(1: a_{1}: \ldots: a_{d}\right) \leq \frac{3}{2} d^{2} h+8 d^{2} \log 2
$$

(utiliser le lemme 3.4, p. 29 de [Da]). En appliquant directement le lemme 2.1, inégalité (ii) et les inégalités p. 18 (resp. p. 27) concernant les quantités notées $e_{1}$ (resp. $e_{2}$ ) dans [Da] $\left({ }^{6}\right)$, nous trouvons que

$$
\max \left\{\left|g_{2}\right|,\left|\wp\left(\omega_{1} / 2\right)\right|,\left|\wp\left(\omega_{2} / 2\right)\right|\right\} \leq \exp \{0.3+2 d h(E)\} .
$$

Cette inégalité implique

$$
\log \max \left\{1,\left|a_{1}\right|, \ldots,\left|a_{d}\right|\right\} \leq 0.3 d+2 d^{2} h .
$$

Nous avons aussi les inégalités analogues évidentes pour les quantités $\wp^{\sharp}\left(\omega_{1}^{\sharp} / 2\right)$ et $\wp^{\sharp}\left(\omega_{2}^{\sharp} / 2\right)$.

Choix de la matrice $\mathcal{B}$ dans le lemme de Thue-Siegel. Notons $\mathcal{M}_{\underline{D}}$ l'ensemble de tous les monômes unitaires $\underline{X}^{\underline{\lambda}}$ qui soient $\mathbb{C}$-linéairement indépendants sur $G$ et tels que $\underline{\lambda} \leq \underline{D}$. Le cardinal de $\mathcal{M}_{\underline{D}}$ est égal à

$$
\frac{1}{4 !} \mathcal{H}(G: \underline{D})=3^{4} \prod_{n} D_{n}
$$

d'après [Da], lemme 6.2, p. 53. Soit $\mathcal{E}_{\underline{D}}$ l'ensemble des quintuplets $\left(j, \lambda_{1}\right.$, $\left.\ldots, \lambda_{4}\right)$ avec $a_{j} X_{1}^{\lambda_{1}} \ldots X_{4}^{\lambda_{4}} \in \mathcal{M}_{\underline{D}}$, et soit $L=3^{4} d \prod_{n} D_{n}$ son cardinal.

Nous posons

$$
\mathcal{B}=\left(\mathcal{B}_{\partial, \nu}\right)_{\substack{\partial \in \mathcal{T}_{T_{0}, 2 T} \\ \nu \in \mathcal{E}_{\underline{D}}}} \in \operatorname{Mat}_{\widetilde{T} \times L}(\mathbb{C}),
$$

où le coefficient $\mathcal{B}_{\partial, \nu}$, pour $\nu=\left(j, \lambda_{1}, \ldots, \lambda_{4}\right)$ est égal à

$$
\left.a_{j}\left(\partial \wp\left(z_{1}\right)^{\lambda_{1}} \wp\left(z_{2}\right)^{\lambda_{2}} \wp^{\sharp}\left(m_{1,1} z_{1}+m_{1,2} z_{2}\right)^{\lambda_{3}} \wp^{\sharp}\left(m_{2,1} z_{1}+m_{2,2} z_{2}\right)^{\lambda_{4}}\right)\right|_{\substack{z_{1} \mapsto \omega_{1} / 2 \\ z_{2} \mapsto \omega_{2} / 2}} .
$$

En choisissant des indexations pour $\mathcal{M}_{\underline{D}}$ et $\mathcal{T}_{T_{0}, 2 T}$ nous avons le système d'inégalités (18) que nous voulons résoudre.

$\left({ }^{6}\right)$ Nous rappelons que dans [Da] on pose $e_{1}=\omega_{1}^{2} \wp\left(\omega_{1} / 2\right)$ et $e_{2}=\omega_{1}^{2} \wp\left(\omega_{2} / 2\right)$. 
Calcul d'un majorant $M$ dans le lemme de Thue-Siegel. Nous devons calculer explicitement un majorant $M$ dans (17). Nous parviendrons à la valeur correspondante au terme de droite dans l'inégalité (22) ci-dessous.

Nous avons besoin du lemme suivant (cf. lemme 3.5, p. 30 de [Da]).

Lemme 3.4. Tout coefficient $\mathcal{B}_{\partial, \nu}$ de la matrice $\mathcal{B}$ introduite plus bas satisfait

$$
\begin{aligned}
\log \max \left\{1,\left|\mathcal{B}_{\partial, \nu}\right|\right\} \leq & d \log \left(\sum_{n} D_{n}+2 T\right)(2 T+23.19+6 h) \\
& +2 T d\left(15.52+3 \log d+\frac{3}{2} \log N+8.17 h\right) \\
& +\frac{3}{2} d^{3} h+8 d^{3} \log 2 .
\end{aligned}
$$

Pour démontrer ce lemme nous devons utiliser le lemme qui suit, variante de la proposition 8.1, p. 111 de [Da].

Lemme 3.5. Soit $P$ un polynôme dans $K[\underline{X}, \underline{Y}]$ de degré total $D$ avec $\underline{X}=X_{1}, \ldots, X_{4}$ et $\underline{Y}=Y_{1}, \ldots, Y_{4}$. Notons $f_{P}\left(z_{1}, z_{2}\right)$ la fonction abélienne de deux variables complexes obtenue en remplaçant $X_{1}, X_{2}, X_{3}, X_{4}$ par $\wp\left(z_{1}\right), \wp\left(z_{2}\right), \wp^{\sharp}\left(m_{1,1} z_{1}+m_{1,2} z_{2}\right), \wp^{\sharp}\left(m_{2,1} z_{1}+m_{2,2} z_{2}\right)$ et $Y_{1}, Y_{2}, Y_{3}, Y_{4}$ par $\wp^{\prime}\left(z_{1}\right), \wp^{\prime}\left(z_{2}\right), \wp^{\sharp \prime}\left(m_{1,1} z_{1}+m_{1,2} z_{2}\right), \wp^{\sharp \prime}\left(m_{2,1} z_{1}+m_{2,2} z_{2}\right)$. La fonction abélienne $g\left(z_{1}, z_{2}\right)=\left(\partial^{\mu_{1}} / \partial z_{1}^{\mu_{1}}\right)\left(\partial^{\mu_{2}} / \partial z_{2}^{\mu_{2}}\right) f_{P}\left(z_{1}, z_{2}\right)$ s'exprime comme un polynôme $P_{1} \in K\left[\underline{X}^{\prime}, \underline{Y}^{\prime}\right]$ (où $\underline{X}^{\prime}, \underline{Y}^{\prime}$ sont deux paquets de huit variables) évalué en les 8 fonctions $\wp\left(z_{1}\right), \ldots, \wp^{\sharp \prime}\left(m_{2,1} z_{1}+m_{2,2} z_{2}\right)$, de degré $D+\mu_{1}+\mu_{2}$ et de hauteur satisfaisant

$$
\begin{aligned}
h\left(P_{1}\right) \leq & \left(\mu_{1}+\mu_{2}\right) \\
& \times\left(\log \left(3^{5} 2^{9}\right)+3 \log \max _{1 \leq i, j \leq 2}\left\{\left|m_{i, j}\right|\right\}+\log \left(D+\mu_{1}+\mu_{2}\right)\right) .
\end{aligned}
$$

De plus $g\left(\omega_{1} / 2, \omega_{2} / 2\right) \in K$ et

$$
\begin{aligned}
h\left(g\left(\omega_{1} / 2, \omega_{2} / 2\right)\right) \leq & h(P)+\log \left(\sum_{n} D_{n}+\mu_{1}+\mu_{2}\right)(23.19+6 h) \\
& +\left(\mu_{1}+\mu_{2}\right)\left(\Xi+\log \left(\sum_{n} D_{n}+\mu_{1}+\mu_{2}\right)\right),
\end{aligned}
$$

où $\Xi=14.42+3 \log d+(3 / 2) \log N+(15 / 2) h$.

Démonstration. La démonstration de l'inégalité (23) est immédiate en utilisant les arguments de [Da], pp. 111-114. On utilise directement l'équation de Weierstrass qui donne $\wp^{\prime \prime}(z)=6 \wp(z)-g_{2} / 2$.

Montrons comment l'inégalité (23) implique l'inégalité (24). Le lemme 10.3, p. 133 de [Da] implique

$$
\max _{1 \leq i, j \leq 2}\left\{\left|m_{i, j}\right|\right\} \leq d\left(6 N h(E) h\left(E^{\sharp}\right)\right)^{1 / 2} .
$$


Donc on peut majorer $h\left(P_{1}\right)$ ainsi :

$$
\begin{aligned}
h\left(P_{1}\right) \leq & h(P)+\left(\mu_{1}+\mu_{2}\right)\left(11.74+3 \log d+\frac{3}{2} \log 6+\frac{3}{2} \log N\right. \\
& \left.+\frac{3}{2} \log h(E)+\frac{3}{2} \log h\left(E^{\sharp}\right)+6 h+\log \left(\sum_{n} D_{n}+\mu_{1}+\mu_{2}\right)\right) \\
\leq & h(P)+\left(\mu_{1}+\mu_{2}\right)\left(\Xi+\log \left(\sum_{n} D_{n}+\mu_{1}+\mu_{2}\right)\right) .
\end{aligned}
$$

Remplaçons $z_{1}$ par $\omega_{1} / 2$ et $z_{2}$ par $\omega_{2} / 2$ dans l'expression trouvée pour $g\left(z_{1}, z_{2}\right)$. Les équations de Weierstrass impliquent $g\left(\omega_{1} / 2, \omega_{2} / 2\right) \in K$. Nous avons de plus

$$
\begin{aligned}
h\left(g\left(\omega_{1} / 2, \omega_{2} / 2\right)\right) \leq & h\left(P_{1}\right)+\log \left(\sum_{n} D_{n}+\mu_{1}+\mu_{2}\right)+\left(\sum_{n} D_{n}+\mu_{1}+\mu_{2}\right) \\
& \times h\left(1: \wp\left(\omega_{1} / 2\right): \wp\left(\omega_{2} / 2\right): \wp^{\sharp}\left(\omega_{1}^{\sharp} / 2\right): \wp^{\sharp}\left(\omega_{2}^{\sharp} / 2\right)\right) \\
\leq & h(P)+\log \left(\sum_{n} D_{n}+\mu_{1}+\mu_{2}\right)(1+32 \log 2+6 h) \\
& +\left(\mu_{1}+\mu_{2}\right)\left(\Xi+\log \left(\sum_{n} D_{n}+\mu_{1}+\mu_{2}\right)\right) .
\end{aligned}
$$

Démonstration du lemme 3.4. Comme la matrice de changement de la base $\left(\partial_{1}, \partial_{2}\right)$ à la base $\left(\left(\partial / \partial z_{1}\right),\left(\partial / \partial z_{2}\right)\right)$ est $\left(\begin{array}{cc}\omega_{1} & \omega_{2} \\ \omega_{2} & -\omega_{1}\end{array}\right)$, on a, d'après le lemme 3.1 de [Phi-Wa],

$$
\begin{aligned}
\left|\partial_{1}^{\mu_{1}} \partial_{2}^{\mu_{2}} f_{P}\left(\omega_{1} / 2, \omega_{2} / 2\right)\right| \leq & \left(\left|\omega_{1}\right|+\left|\omega_{2}\right|\right)^{\mu_{1}+\mu_{2}}\left|\frac{\partial^{\mu_{1}}}{\partial z_{1}^{\mu_{1}}} \frac{\partial^{\mu_{2}}}{\partial z_{2}^{\mu_{2}}} f_{P}\left(\omega_{1} / 2, \omega_{2} / 2\right)\right| \\
\leq & \exp \left\{(2.2+(2 / 3) d h(E))\left(\mu_{1}+\mu_{2}\right)\right\} \\
& \times\left|\frac{\partial^{\mu_{1}}}{\partial z_{1}^{\mu_{1}}} \frac{\partial^{\mu_{2}}}{\partial z_{2}^{\mu_{2}}} f_{P}\left(\omega_{1} / 2, \omega_{2} / 2\right)\right|,
\end{aligned}
$$

où $f_{P}\left(z_{1}, z_{2}\right)$ est la fonction abélienne du lemme 3.5 correspondant au polynôme $P=a_{j} X_{1}^{\lambda_{1}} X_{2}^{\lambda_{2}} X_{3}^{\lambda_{3}} X_{4}^{\lambda_{4}}$ où $1 \leq j \leq d$ et $\underline{\lambda} \leq \underline{D}$. La dernière inégalité est obtenue en appliquant le lemme 2.1.

Pour majorer $\left|g\left(\omega_{1} / 2, \omega_{2} / 2\right)\right|$ il suffit d'utiliser le lemme 3.5. L'inégalité (24) implique

$$
\begin{aligned}
h\left(g\left(\omega_{1} / 2, \omega_{2} / 2\right)\right) \leq & (3 / 2) d^{2} h+8 d^{2} \log 2 \\
& +\log \left(\sum_{n} D_{n}+\mu_{1}+\mu_{2}\right)(23.19+6 h) \\
& +\left(\mu_{1}+\mu_{2}\right)\left(\Xi+\log \left(\sum_{n} D_{n}+\mu_{1}+\mu_{2}\right)\right),
\end{aligned}
$$

car $h(P) \leq h\left(1: a_{j}\right) \leq h\left(1: a_{1}: \ldots: a_{d}\right)$ d'après l'inégalité (19). Comme 
$\log \left|g\left(\omega_{1} / 2, \omega_{2} / 2\right)\right| \leq d h\left(g\left(\omega_{1} / 2, \omega_{2} / 2\right)\right)$, nous avons prouvé l'inégalité (22) et la démonstration du lemme 3.4 est complète.

Estimation de $\varsigma$. Soit $\varepsilon$ un nombre réel comme dans le sous-paragraphe 3.1.

Lemme 3.6. Avec notre choix de paramètres (10), le rang $\varsigma$ de la matrice $\mathcal{B}$ est majoré par la quantité suivante :

$$
\begin{aligned}
\varsigma & \leq(1+3 \varepsilon) 4 ! \cdot 3^{5} \cdot 2^{6} \prod_{n} D_{n} \frac{T_{0}\left(2 T-T_{0}\right)}{T^{2}} \\
& \leq 373260 C_{1}^{4} C_{2}^{-2} C_{3}\left(2 C_{2}-C_{3}\right) d^{4}(\log \max \{e, d\})^{4} \mathcal{L}^{4} .
\end{aligned}
$$

Remarquons immédiatement que cette majoration ne dépend pas de $\mathcal{V}$.

Démonstration. On suit les calculs explicites de la démonstration du lemme 6.1, pp. 47-53 de [Da]. Après avoir observé dans le lemme 3.1 que la sous-variété abélienne notée $\mathfrak{H}_{0}$ est telle que $\varrho_{0}=1$ (resp. $\varrho_{0}=2$ ), et que $\operatorname{dim}\left(\mathfrak{H}_{0}\right)=2$ (resp. 0), appliquons les calculs de [Da], p. 50, pour estimer le rang $\varsigma$. Nous avons

$$
\varsigma \leq \xi_{0}\left(\operatorname{dim} \mathfrak{H}_{0}+1\right) \mathcal{H}\left(\mathfrak{H}_{0}: \underline{D}\right),
$$

où $\xi_{0}$ est égal au cardinal de l'ensemble $\mathcal{T}_{T_{0}, 2 T}$ si $\varrho_{0}=2$, ou égal à $T_{0}+1$ si $\varrho_{0}=1$ (ce calcul est aisément déduit de la situation plus générale abordée p. 48 loc. cit.). Plus concrètement nous avons

$$
\xi_{0}= \begin{cases}(1 / 2)\left(T_{0}+1\right)\left(2 T+2-T_{0}\right) & \text { si } \varrho_{0}=2 \\ T_{0}+1 & \text { si } \varrho_{0}=1 .\end{cases}
$$

D'après le lemme 3.1 , identité (14) et l'expression $\mathcal{H}(G: \underline{D})=3^{4} \cdot 4$ ! $\prod_{n} D_{n}$, on a

$$
\begin{aligned}
\mathcal{H}\left(\mathfrak{H}_{0}: \underline{D}\right) & =(1+\varepsilon) \frac{3^{4} \cdot 4 ! \prod_{n} D_{n}}{\left([(T-1) / 4]+\varrho_{0}\right)} \\
& \leq(1+\varepsilon) 3^{4} \cdot 4 ! \cdot 4 \cdot \prod_{n} D_{n} \begin{cases}\frac{4}{T-5} & \text { si } \varrho_{0}=1, \\
\frac{2^{5}}{(T-1)(T-3)} & \text { si } \varrho_{0}=2 .\end{cases}
\end{aligned}
$$

En remplaçant ces estimations dans l'inégalité (26), on obtient que la majoration la plus défavorable (celle qu'il faut donc retenir) intervient pour $\varrho_{0}=1$ et dans ce cas $\operatorname{dim}\left(\mathfrak{H}_{0}\right)=2$. Avec notre choix des paramètres (10) nous vérifions que nos estimations ne dépendent pas de $\mathcal{V}$, et nous obtenons précisément l'inégalité (25); la preuve du lemme 3.6 est terminée.

Vérification des conditions dans le lemme de Thue-Siegel. Terminons la preuve du lemme 3.2. Il s'agit, avec notre choix de paramètres et la majo- 
ration trouvée pour le rang $\varsigma$, de vérifier l'inégalité (16). Nous avons déjà souligné que cette majoration ne dépend pas de la quantité $\mathcal{V}$.

Nous avons $d \geq 2$ et nous avons aussi $\mathcal{L}>h \geq 4, \varepsilon<10^{-5}$; nous appliquons l'encadrement (9), justifié par l'encadrement (12), qui permet de majorer tous les termes positifs proportionnels à $\log \mathcal{V}$ et $\mathcal{V}^{-1}$.

Sur la base du choix des paramètres (10), du lemme 3.4 et du lemme 3.6, on a les majorations

$$
\begin{aligned}
2 \varsigma & \log \left[2 \widetilde{T} e^{Q+M+U}+1\right] \\
\leq & 373260 C_{1}^{4} C_{2}^{-2} C_{3}\left(2 C_{2}-C_{3}\right)\left(C_{6} / 2+C_{5}+\left(2.31 \log C_{1}+36.28\right) C_{2}\right. \\
& \left.+4 \cdot 10^{-4}\left(4 \log C_{1}+\log C_{3}\right)\right) d^{7}(\log \max \{e, d\})^{7} \mathcal{L}^{7} \mathcal{V} \\
\leq & 2 \cdot 10^{113} d^{7}(\log \max \{e, d\})^{7} \mathcal{L}^{7} \mathcal{V} .
\end{aligned}
$$

De même on trouve les minorations suivantes :

$$
L Q \geq 3{ }^{4} C_{6} C_{1}^{4} d^{7}(\log \max \{e, d\})^{7} \mathcal{L}^{7} \mathcal{V} \geq 10^{114} d^{7}(\log \max \{e, d\})^{7} \mathcal{L}^{7} \mathcal{V}
$$

Ainsi la condition (16) est satisfaite et nous pouvons construire un polynôme $P$ satisfaisant toutes les propriétés du lemme 3.2. En particulier la majoration des valeurs absolues de ses coefficients provient de l'inégalité (21) combinée avec la majoration $\max \left\{\left|p_{i}\right|\right\} \leq e^{Q}$ du lemme 3.3. Pour montrer la majoration de la hauteur de $P$ il suffit d'appliquer l'inégalité (19). On a

$$
\begin{aligned}
h(P) & \leq h\left(1: a_{1}: \ldots: a_{d}\right)+\log \max \left\{\left|p_{j}\right|\right\}+\log d \\
& \leq(3 / 2) d^{2} h+8 d^{2} \log 2+Q+\log d .
\end{aligned}
$$

La preuve du lemme 3.2 est complète.

3.3. Deuxième pas : extrapolation. Nous allons maintenant prouver que la fonction $f_{P}$ du lemme 3.2 s'annule à un ordre $\geq T$ en $\left(\omega_{1} / 2, \omega_{2} / 2\right)$. Nous allons établir essentiellement un lemme de croissance analytique et une minoration arithmétique. La conclusion vient d'un lemme de Schwarz approché en une variable complexe. Le résultat original de ce paragraphe est le lemme 3.7.

Croissance analytique. Notons

$$
z_{1}^{\sharp}=z_{1}^{\sharp}\left(z_{1}, z_{2}\right)=m_{1,1} z_{1}+m_{1,2} z_{2} \quad \text { et } \quad z_{2}^{\sharp}=z_{2}^{\sharp}\left(z_{1}, z_{2}\right)=m_{2,1} z_{1}+m_{2,2} z_{2},
$$

pour deux variables complexes $z_{1}$ et $z_{2}$. Notons $\eta_{1}, \eta_{2}$ (resp. $\eta_{1}^{\sharp}, \eta_{2}^{\sharp}$ ) les quasipériodes fondamentales associées au réseau $\Lambda$ (resp. $\Lambda^{\sharp}$ ) (cf. [Lan]). Posons

$$
\Theta\left(z_{1}, z_{2}\right)=\varphi\left(z_{1}\right)^{3 D_{1}} \varphi\left(z_{2}\right)^{3 D_{2}} \varphi^{\sharp}\left(z_{1}^{\sharp}\right)^{3 D_{3}} \varphi^{\sharp}\left(z_{2}^{\sharp}\right)^{3 D_{4}},
$$

où $\varphi$ désigne la fonction associée au réseau $\Lambda$ définie par

$$
\varphi(z)=\exp \left\{-\frac{\eta_{2}}{2 \omega_{1}}\left(\frac{z}{\omega_{1}}\right)^{2}\right\} \exp \left\{i \pi \frac{z}{\omega_{1}}\right\} \sigma(z),
$$


et $\varphi^{\sharp}$ est la fonction associée au réseau $\Lambda^{\sharp}$ :

$$
\varphi^{\sharp}(z)=\exp \left\{-\frac{\eta_{2}^{\sharp}}{2 \omega_{1}^{\sharp}}\left(\frac{z}{\omega_{1}^{\sharp}}\right)^{2}\right\} \exp \left\{i \pi \frac{z}{\omega_{1}^{\sharp}}\right\} \sigma^{\sharp}(z) .
$$

On a, d'après [Da], p. 13, les formules suivantes qui permettent de passer aux fonctions de Weierstrass normalisées $\widetilde{\varphi}$ et $\widetilde{\wp}$ (i.e. relatives au réseau $\Lambda / \omega_{1}$; on a des formules analogues pour le réseau $\left.\Lambda^{\sharp} / \omega_{1}^{\sharp}\right)$ :

$$
\varphi\left(z_{j}\right)^{3}=\omega_{1}^{3} \widetilde{\varphi}\left(\frac{z_{j}}{\omega_{1}}\right)^{3}, \quad \varphi\left(z_{j}\right)^{3} \wp\left(z_{j}\right)=\omega_{1} \widetilde{\varphi}\left(\frac{z_{j}}{\omega_{1}}\right)^{3} \widetilde{\wp}\left(\frac{z_{j}}{\omega_{1}}\right) .
$$

Soit $P \in \mathbb{C}[\underline{X}]$ de multidegré $\leq \underline{D}=\left(D_{1}, \ldots, D_{4}\right)$, soit $f_{P}$ la fonction abélienne $\mathbb{C}^{2} / \Lambda^{2} \rightarrow \mathbb{C}$ introduite dans l'énoncé de la proposition 3.1. Soit $F_{P}: \mathbb{C}^{2} \rightarrow \mathbb{C}$ la fonction analytique de deux variables complexes

$$
F_{P}\left(z_{1}, z_{2}\right)=\Theta\left(z_{1}, z_{2}\right) f_{P}\left(z_{1}, z_{2}\right) .
$$

Nous allons étudier la croissance analytique de la fonction entière $G(z)=$ $F_{P}\left(\omega_{1} z, \omega_{2} z\right)$. Précisons que le lemme qui suit est d'importance fondamentale pour la preuve de nos résultats; les raisons ont été déjà expliquées dans le sous-paragraphe 1.2.

Lemme 3.7. Fixons un polynôme $P \in \mathbb{C}[\underline{X}]$ de multidegré $\leq \underline{D}$ dont la valeur absolue des coefficients est majorée par $e^{H}$ pour un nombre réel positif $H$ et considérons $F_{P}$ comme ci-dessus.

Soient $\mu_{1}$ et $\mu_{2}$ deux entiers positifs, soit $z$ un nombre complexe. On a

$$
\begin{gathered}
\left|\left(\partial_{1}^{\mu_{1}} \partial_{2}^{\mu_{2}} F_{P}\right)\left(\omega_{1} z, \omega_{2} z\right)\right| \leq e^{H} M_{1}, \\
\left|\left(\partial_{1}^{\mu_{1}} \partial_{2}^{\mu_{2}} \Theta\right)\left(\omega_{1} z, \omega_{2} z\right)\right| \leq M_{1},
\end{gathered}
$$

où nous avons posé $M_{1}=a(|z|)+b\left(\mu_{1}+\mu_{2}\right)$ avec

$$
\begin{aligned}
a(u)= & \left(\sum_{n} D_{n}\right)(143+80.4 d h+67.1 u)+3 \pi^{2} \sum_{n} D_{n} \zeta_{n}^{*} \\
& +3 \pi(1+3 \sqrt{3}) u \sum_{n} D_{n} \zeta_{n}, \\
b(v)= & v\left(1.4+2 \log d+(1 / 2) \log N+(3 / 2) \log h(E)+(1 / 2) \log h\left(E^{\sharp}\right)\right. \\
& +\log (v+1)),
\end{aligned}
$$

et nous avons posé $\left(\zeta_{1}^{*}, \zeta_{2}^{*}, \zeta_{3}^{*}, \zeta_{4}^{*}\right):=\left(y^{-1}, y+1+\sqrt{3} / 6, y^{\sharp-1}, y^{\sharp}+1+\sqrt{3} / 6\right)$.

Démonstration. Introduisons des variables complexes $s_{1}$ et $s_{2}$ satisfaisant

$$
z_{1}=\omega_{1} s_{1}+\omega_{2} s_{2}, \quad z_{2}=\omega_{2} s_{1}-\omega_{1} s_{2} .
$$

Pour toute fonction analytique $A\left(z_{1}, z_{2}\right)$ de deux variables complexes on a

$$
\partial_{1}^{\mu_{1}} \partial_{2}^{\mu_{2}} A\left(z_{1}, z_{2}\right)=\left(\frac{\partial}{\partial s_{1}}\right)^{\mu_{1}}\left(\frac{\partial}{\partial s_{2}}\right)^{\mu_{2}} A\left(\omega_{1} s_{1}+\omega_{2} s_{2}, \omega_{2} s_{1}-\omega_{1} s_{2}\right) .
$$


Nous démontrons l'inégalité (27) dans le cas particulier de $\mu_{1}=\mu_{2}=0$. Nous appliquons ensuite l'inégalité de Cauchy pour en déduire le cas général. Fixons $z \in \mathbb{C}$ et estimons la fonction $\left|F_{P}\left(\omega_{1} s_{1}+\omega_{2} s_{2}, \omega_{2} s_{1}-\omega_{1} s_{2}\right)\right|$ pour tout $\left(s_{1}, s_{2}\right) \in \gamma_{1} \times \gamma_{2}$ avec $\gamma_{1}=\left\{s_{1}^{\prime} \in \mathbb{C}:\left|s_{1}^{\prime}-z\right|=\delta\right\}$ et $\gamma_{2}=\left\{s_{2}^{\prime} \in \mathbb{C}:\left|s_{2}^{\prime}\right|=\delta\right\}$, où $\delta$ est un nombre réel positif que nous allons fixer un peu plus bas $\left(\gamma_{1} \times \gamma_{2}\right.$ est le bord du polydisque de centre $(z, 0)$ et rayon $\delta$ ).

Nous avons $\left|\Im\left(z_{1} / \omega_{1}\right)\right| \leq \delta(y+3 / 2)+|z|$ et $\left|\Im\left(z_{2} / \omega_{1}\right)\right| \leq(|z|+\delta)(y+$ $1 / 2)+\delta$ pour $\left(z_{1}, z_{2}\right)$ et $\left(s_{1}, s_{2}\right)$ liés par $(29)$ et $\left(s_{1}, s_{2}\right) \in \gamma_{1} \times \gamma_{2}$.

Nous fixons une fois pour toutes la valeur suivante de $\delta$ :

$$
\delta:=\max \left\{\left|m_{1,1} \tau-m_{1,2}\right|,\left|m_{2,1} \tau-m_{2,2}\right|\right\}^{-1} .
$$

Posons

$$
\omega_{1}^{*}=m_{1,1} \omega_{2}-m_{1,2} \omega_{1}, \quad \omega_{2}^{*}=m_{2,1} \omega_{2}-m_{2,2} \omega_{1} .
$$

Pour commencer montrons que l'on a les estimations suivantes :

$$
d^{-1}\left(6 N h(E) h\left(E^{\sharp}\right)\right)^{-1 / 2}(1+(3 / 4) d h(E))^{-1} \leq \delta,
$$

(ii) $\delta \leq 2 \sqrt{3} / 3$,

$$
\begin{aligned}
& \text { (iii) } \delta \max \left\{\left|\frac{\omega_{1}^{*}}{\omega_{1}^{\sharp}}\right|,\left|\frac{\omega_{2}^{*}}{\omega_{1}^{\sharp}}\right|\right\} \leq \frac{2^{1 / 2}}{3^{1 / 4}} N^{-1 / 2} y^{\sharp 1 / 2}, \\
& \text { (iv) } \delta^{2} \max \left\{\left|\frac{\omega_{1}^{*}}{\omega_{1}^{\sharp}}\right|,\left|\frac{\omega_{2}^{*}}{\omega_{1}^{\sharp}}\right|\right\} / y^{\sharp} \leq \frac{2 \sqrt{3}}{3} N^{-1} .
\end{aligned}
$$

(i) On a $\delta^{-1}=\max \left\{\left|m_{1,1} \tau-m_{1,2}\right|,\left|m_{2,1} \tau-m_{2,2}\right|\right\}$, que nous allons majorer. Comme

$$
\delta^{-1} \leq \max _{i, j}\left\{\left|m_{i, j}\right|\right\}(1+|\tau|),
$$

on peut utiliser le lemme 10.3, p. 133 de [Da] et la première inégalité du lemme 2.1 .

(ii) On a $\delta \leq\left|m_{1,1} \tau-m_{1,2}\right|^{-1}$. Écrivons $\tau=x+i y$ et observons que $\left|m_{1,1} \tau-m_{1,2}\right|=\left(\left(m_{1,1} x-m_{1,2}\right)^{2}+\left(m_{1,1} y\right)^{2}\right)^{1 / 2}$, que l'on veut minorer. On a deux cas selon que $m_{1,1}=0$ ou non. Supposons d'abord $m_{1,1}=0$. On a donc $m_{1,2} \neq 0$ et $\delta \leq 1$. Si $m_{1,1} \neq 0$ alors

$$
\delta \leq\left(\left(m_{1,1} x-m_{1,2}\right)^{2}+\left(m_{1,1} y\right)^{2}\right)^{-1 / 2} \leq\left(\left|m_{1,1}\right| y\right)^{-1} \leq 2 \sqrt{3} / 3 .
$$

On trouve $\delta \leq \max \{1,2 \sqrt{3} / 3\}=2 \sqrt{3} / 3$ et on termine la vérification de (ii).

(iii) On a

$$
\delta \max \left\{\left|\frac{\omega_{1}^{*}}{\omega_{1}^{\sharp}}\right|,\left|\frac{\omega_{2}^{*}}{\omega_{1}^{\sharp}}\right|\right\} \leq\left|m_{1,1}+m_{1,2} \tau\right|^{-1} .
$$

D'après les formules de [Da], p. 134 on a

$$
\left|m_{1,1}+m_{1,2} \tau\right|=N^{1 / 2} \frac{y^{1 / 2}}{y^{\sharp 1 / 2}} \geq \frac{3^{1 / 4}}{2^{1 / 2}} N^{1 / 2} y^{\sharp-1 / 2} .
$$


(iv) On a

$$
\delta^{2} \max \left\{\left|\frac{\omega_{1}^{*}}{\omega_{1}^{\sharp}}\right|,\left|\frac{\omega_{2}^{*}}{\omega_{1}^{\sharp}}\right|\right\}^{2} / y^{\sharp} \leq\left|m_{1,1}+m_{1,2} \tau\right|^{-2} / y^{\sharp} .
$$

Comme $\left|m_{1,1}+m_{1,2} \tau\right|^{-2} / y^{\sharp}=N^{-1} y^{-1}$, la dernière inégalité en découle.

Introduisons quatre indéterminées $Y_{i}, i=1, \ldots, 4$. On a

$$
\underline{Y}^{\underline{D}} P(\underline{X})=\sum_{\underline{\lambda}}(\underline{X Y})^{\underline{\lambda}} \underline{Y}^{\underline{D}-\underline{\lambda}} .
$$

On peut voir $F_{P}$ comme polynôme en huit variables évalué en $\varphi\left(z_{j}\right)^{3}$, $\varphi\left(z_{j}\right)^{3} \wp\left(z_{j}\right)$ et les fonctions analogues associées au réseau $\Lambda^{\sharp}$, de multidegré $\leq \underline{D}$ (cette fois en quatre paquets de deux variables correspondant à $\underline{Y}$ et $\underline{X Y})$.

Nous appliquons les majorations pour $\left|\phi_{\tau}(z)\right|^{3}$ (resp. $\left.\left|\phi_{\tau}(z)^{3} \wp_{\tau}(z)\right|\right)$ qui interviennent p. 17 (resp. p. 19) de [Da] (notations de l'auteur) :

$$
\begin{aligned}
\max \left\{\left|\widetilde{\varphi}\left(\frac{z_{1}}{\omega_{1}}\right)^{3}\right|\right. & \left.,\left|\widetilde{\varphi}\left(\frac{z_{1}}{\omega_{1}}\right)^{3} \widetilde{\wp}\left(\frac{z_{1}}{\omega_{1}}\right)\right|\right\} \\
\leq & 0.309 \exp \left\{3 \pi \left(|z|^{2} / y+3|z| \delta / y+|z|(2 \delta+1)\right.\right. \\
& \left.\left.+y\left(\delta^{2}+\delta+1 / 4\right)+3 \delta^{2}+9 \delta^{2} /(4 y)+3 \delta / 2\right)\right\} \\
\leq & \exp \left\{3 \pi\left(|z|^{2}+2 \sqrt{3}|z|\right) / y+31.2|z|+25.9 y+85.6\right\}
\end{aligned}
$$

Pour prouver ces estimations nous avons utilisé les majorations données dans (30)(ii) et la minoration $y \geq \sqrt{3} / 2$. Comme nous allons les utiliser très souvent nous ne les mentionnerons qu'occasionnellement.

De la même manière on tire

$$
\begin{aligned}
& \max \left\{\left|\widetilde{\varphi}\left(\frac{z_{2}}{\omega_{1}}\right)^{3}\right|,\left|\widetilde{\varphi}\left(\frac{z_{2}}{\omega_{1}}\right)^{3} \widetilde{\wp}\left(\frac{z_{2}}{\omega_{1}}\right)\right|\right\} \\
& \leq 0.309 \exp \left\{3 \pi \left(|z|^{2}(y+1+1 /(4 y))+|z| y(2 \delta+1)\right.\right. \\
&+|z|(4 \delta+1 / 2+3 \delta /(2 y)) \\
&\left.\left.+\left(\delta^{2} y+\delta y+y / 4\right)+\left(3 \delta^{2}+9 \delta^{2} /(4 y)+3 /(2 \delta)\right)\right)\right\} \\
& \leq \exp \left\{3 \pi\left(|z|^{2}(y+1+1 /(4 y))+(3+4 \sqrt{3}) y|z| / 3\right)\right. \\
&+67.1|z|+25.9 y+85.5\} \\
& \leq \exp \left\{3 \pi\left(|z|^{2}(y+1+\sqrt{3} / 6)+(3+4 \sqrt{3}) y|z| / 3\right)\right. \\
&+67.1|z|+25.9 y+85.5\} .
\end{aligned}
$$

Cela se vérifie sans peine comme l'inégalité précédente. Pour les estimations des fonctions de Weierstrass relatives au réseau $\Lambda^{\sharp}$ on remarque que $z_{1}^{\sharp}=$ $s_{1} \omega_{1}^{\sharp}-s_{2} \omega_{1}^{*}$ et $z_{2}^{\sharp}=s_{1} \omega_{2}^{\sharp}-s_{2} \omega_{2}^{*}$. On obtient donc 


$$
\begin{aligned}
& \max \left\{\left|\tilde{\varphi}^{\sharp}\left(\frac{z_{1}^{\sharp}}{\omega_{1}^{\sharp}}\right)^{3}\right|,\left|\tilde{\varphi}^{\sharp}\left(\frac{z_{1}^{\sharp}}{\omega_{1}^{\sharp}}\right)^{3} \widetilde{\rho}^{\sharp}\left(\frac{z_{1}^{\sharp}}{\omega_{1}^{\sharp}}\right)\right|\right\} \\
& \leq 0.309 \exp \left\{3 \pi \left(\frac{|z|^{2}}{y^{\sharp}}+\frac{2|z| \delta}{y^{\sharp}}+|z|\left(1+\frac{2 \delta}{y^{\sharp}}\left|\frac{\omega_{1}^{*}}{\omega_{1}^{\sharp}}\right|\right)\right.\right. \\
&\left.\left.+\left(\delta+\frac{\delta^{2}}{y^{\sharp}}+\frac{2 \delta^{2}}{y^{\sharp}}\left|\frac{\omega_{1}^{*}}{\omega_{1}^{\sharp}}\right|+\frac{\delta^{2}}{y^{\sharp}}\left|\frac{\omega_{1}^{*}}{\omega_{1}^{\sharp}}\right|\right)+\left(\delta\left|\frac{\omega_{1}^{*}}{\omega_{1}^{\sharp}}\right|+\frac{y^{\sharp}}{4}\right)\right)\right\} \\
& \leq \exp \left\{3 \pi\left(\frac{|z|^{2}}{y^{\sharp}}+\frac{4 \sqrt{3}}{3} \cdot \frac{|z|}{y^{\sharp}}\right)+31.2|z|+56.7+14.1 y^{\sharp}\right\} .
\end{aligned}
$$

Ici, en utilisant les estimations données dans (30), en particulier (ii), (iii) et (iv), nous avons remarqué que $\left|\omega_{1}^{*} / \omega_{1}^{\sharp}\right| \delta / y^{\sharp} \leq 2 \sqrt{3} / 3$, que l'on a aussi

$$
\left|\omega_{1}^{*} / \omega_{1}^{\sharp}\right|^{2} \delta^{2} / y^{\sharp} \leq 2 \sqrt{3} / 3 \quad \text { et }\left|\omega_{1}^{*} / \omega_{1}^{\sharp}\right| \delta \leq\left(2^{1 / 2} / 3^{1 / 4}\right)(2 \sqrt{3} / 3) y^{\sharp} .
$$

De la même façon on tire

$$
\begin{aligned}
\max \left\{\left|\widetilde{\varphi}^{\sharp}\left(\frac{z_{2}^{\sharp}}{\omega_{1}^{\sharp}}\right)^{3}\right|,\left|\widetilde{\varphi}^{\sharp}\left(\frac{z_{2}^{\sharp}}{\omega_{1}^{\sharp}}\right)^{3} \widetilde{\varsigma}^{\sharp}\left(\frac{z_{2}^{\sharp}}{\omega_{1}^{\sharp}}\right)\right|\right\} \\
\leq \exp \left\{3 \pi\left(|z|^{2}\left(y^{\sharp}+1+\frac{1}{4 y^{\sharp}}\right)+|z| y^{\sharp}(1+3 \sqrt{3})\right)\right. \\
\left.+43.7|z|+53.1 y^{\sharp}+140\right\} \\
\leq \exp \left\{3 \pi\left(|z|^{2}\left(y^{\sharp}+1+\frac{\sqrt{3}}{6}\right)+|z| y^{\sharp}(1+3 \sqrt{3})\right)\right. \\
\left.+43.7|z|+53.1 y^{\sharp}+140\right\} .
\end{aligned}
$$

Si $P$ est un polynôme comme dans l'énoncé du lemme, $\left(z_{1}, z_{2}\right)$ et si $\left(s_{1}, s_{2}\right)$ sont liés par $(29)$ et $\left(s_{1}, s_{2}\right) \in \gamma_{1} \times \gamma_{2}$, alors

$$
\begin{aligned}
\left|F_{P}\left(z_{1}, z_{2}\right)\right| \leq & e^{H}\left(\max \left\{\left|\omega_{1}\right|,\left|\omega_{1}\right|^{3},\left|\omega_{1}^{\sharp}\right|,\left|\omega_{1}^{\sharp}\right|^{3}\right\}\right)^{\sum_{n} D_{n}} \\
& \times \exp \left\{D_{1}(88.6+39.6 d h(E)+31.2|z|)\right. \\
& +3 \pi D_{1}\left(|z|^{2}+2 \sqrt{3}|z|\right) / y \\
& +D_{2}(88.5+39.6 d h(E)+67.1|z|) \\
& +3 \pi D_{2}\left((y+1+\sqrt{3} / 6)|z|^{2}+(3+4 \sqrt{3}) y|z| / 3\right) \\
& +D_{3}\left(56.7+21.9 d h\left(E^{\sharp}\right)+31.2|z|\right) \\
& +3 \pi D_{3}\left(|z|^{2}+4 \sqrt{3}|z|\right) / y^{\sharp} \\
& +D_{4}\left(143+80.4 d h\left(E^{\sharp}\right)+43.7|z|\right) \\
& \left.+3 \pi D_{4}\left(\left(y^{\sharp}+1+\sqrt{3} / 6\right)|z|^{2}+(1+3 \sqrt{3}) y^{\sharp}|z|\right)\right\}
\end{aligned}
$$




$$
\begin{aligned}
\leq & e^{H} \exp \left\{\left(\sum_{n} D_{n}\right)(143+80.4 d h+67.1|z|)\right. \\
& +3 \pi|z|^{2} \sum_{n} D_{n} \zeta_{n}^{*}+3 \pi(1+3 \sqrt{3})|z| \sum_{n} D_{n} \zeta_{n} .
\end{aligned}
$$

Rappelons que nous avons utilisé la majoration du point (ii) du lemme 2.1 pour estimer le facteur dépendant des $\left|\omega_{1}\right|$ et $\left|\omega_{1}^{\sharp}\right|$.

Pour démontrer le lemme dans le cas général on applique la formule de Cauchy

$$
\begin{aligned}
\partial_{1}^{\mu_{1}} \partial_{2}^{\mu_{2}} F_{P}\left(z_{1}, z_{2}\right) \mid \begin{array}{l}
z_{1} \mapsto \omega_{1} z \\
z_{2} \mapsto \omega_{2} z
\end{array} \\
=\frac{\mu_{1} ! \mu_{2} !}{4 \pi^{2}} \iint_{\gamma_{1}} \frac{F_{P}\left(\omega_{1} s_{1}^{\prime}+\omega_{2} s_{2}^{\prime}, \omega_{2} s_{1}^{\prime}-\omega_{1} s_{2}^{\prime}\right)}{\left(s_{1}^{\prime}-z\right)^{\mu_{1}+1} s_{2}^{\prime \mu_{2}+1}} d s_{1}^{\prime} d s_{2}^{\prime} .
\end{aligned}
$$

Posons $\widetilde{F}_{P}\left(s_{1}, s_{2}\right)=F_{P}\left(\omega_{1} s_{1}+\omega_{2} s_{2}, \omega_{2} s_{1}-\omega_{1} s_{2}\right)$. En appliquant $(30)(\mathrm{i})$ et $(31)$,

$$
\begin{aligned}
\left|\left(\partial_{1}^{\mu_{1}} \partial_{2}^{\mu_{2}} F_{P}\right)\left(\omega_{1} z, \omega_{2} z\right)\right| & \max _{\left(s_{1}, s_{2}\right) \in \gamma_{1} \times \gamma_{2}}\left|\widetilde{F}_{P}\left(s_{1}, s_{2}\right)\right| \mu_{1} ! \mu_{2} ! \delta^{-\mu_{1}-\mu_{2}} \\
\leq & e^{H} \exp \left\{\left(\sum_{n} D_{n}\right)(143+80.4 d h+67.1|z|)\right. \\
& +3 \pi|z|^{2} \sum_{n} D_{n} \zeta_{n}^{*}+3 \pi(1+3 \sqrt{3})|z| \sum_{n} D_{n} \zeta_{n} \\
& +\left(\mu_{1}+\mu_{2}\right)(1.4+2 \log d+(1 / 2) \log N \\
& \left.\left.+(3 / 2) \log h(E)+(1 / 2) \log h\left(E^{\sharp}\right)+\log \left(\mu_{1}+\mu_{2}+1\right)\right)\right\} .
\end{aligned}
$$

Pour démontrer l'inégalité (28), on procède de manière tout à fait analogue. On remarque que pour tout couple $\left(z_{1}, z_{2}\right) \in \mathbb{C}^{2}$,

$\Theta\left(z_{1}, z_{2}\right)$

$$
=\omega_{1}^{3\left(D_{1}+D_{2}\right)} \omega_{1}^{\sharp 3\left(D_{3}+D_{4}\right)} \widetilde{\varphi}\left(\frac{z_{1}}{\omega_{1}}\right)^{3 D_{1}} \widetilde{\varphi}\left(\frac{z_{2}}{\omega_{1}}\right)^{3 D_{2}} \widetilde{\varphi}^{\sharp}\left(\frac{z_{1}^{\sharp}}{\omega_{1}^{\sharp}}\right)^{3 D_{3}} \widetilde{\varphi}^{\sharp}\left(\frac{z_{2}^{\sharp}}{\omega_{1}^{\sharp}}\right)^{3 D_{4}} .
$$

Si l'on considère un couple $\left(s_{1}, s_{2}\right) \in \gamma_{1} \times \gamma_{2}$, nous remarquons, en utilisant la majoration donnée p. 17 de [Da], que la preuve procède de manière tout à fait analogue en remplaçant $H$ par 0 dans la démonstration que nous venons de compléter (nous avons même une petite amélioration, du fait que la constante multiplicative 0.0176 est plus favorable que 0.309 , mais cela n'affecte que des termes secondaires). Le lemme est démontré.

Minoration arithmétique. Nous voulons démontrer le lemme suivant. 
Lemme 3.8. Le nombre $\Theta\left(\omega_{1} / 2, \omega_{2} / 2\right)$ est non nul et vérifie

$$
\log \left|\Theta\left(\omega_{1} / 2, \omega_{2} / 2\right)\right| \geq-\left(\sum_{n} D_{n}\right)(7.47+20.69 d h) .
$$

Supposons qu'il existe deux entiers positifs $\mu_{1}, \mu_{2}$ avec $\mu_{1}+\mu_{2} \leq T$ et tels que $g\left(\omega_{1} / 2, \omega_{2} / 2\right)=\left(\partial / \partial z_{1}\right)^{\mu_{1}}\left(\partial / \partial z_{2}\right)^{\mu_{2}} f_{P}\left(\omega_{1} / 2, \omega_{2} / 2\right) \neq 0$ et supposons que $\mu_{1}+\mu_{2}$ soit minimal avec $g\left(\omega_{1} / 2, \omega_{2} / 2\right) \neq 0$, où $P$ est le polynôme construit dans le lemme 3.2 et $f_{P}$ est la fonction abélienne associée (cf. notations du lemme 3.5$)$. Alors $g\left(\omega_{1} / 2, \omega_{2} / 2\right) \in K$ et

$$
\log \left|g\left(\omega_{1} / 2, \omega_{2} / 2\right)\right| \geq-M_{2}
$$

où nous avons posé

$$
\begin{aligned}
M_{2}= & (3 / 2) d^{3} h+8 d^{3} \log 2+d Q+d \log d \\
& +d \log \left(\sum_{n} D_{n}+T\right)(23.19+6 h) \\
& +d T\left(14.42+3 \log d+(3 / 2) \log N+(15 / 2) h+\log \left(\sum_{n} D_{n}+T\right)\right) .
\end{aligned}
$$

De plus $\partial_{1}^{\mu_{1}} \partial_{2}^{\mu_{2}} F_{P}\left(\omega_{1} / 2, \omega_{2} / 2\right) \neq 0$ et

$$
\log \max _{\mu_{1}^{\prime}+\mu_{2}^{\prime}=\mu_{1}+\mu_{2}}\left|\partial_{1}^{\mu_{1}^{\prime}} \partial_{2}^{\mu_{2}^{\prime}} F_{P}\left(\omega_{1} / 2, \omega_{2} / 2\right)\right| \geq-M_{3}
$$

où nous avons posé

$$
\begin{aligned}
M_{3}= & (3 / 2) d^{3} h+8 d^{3} \log 2+d Q+d \log d \\
& +d T\left(14.42+3 \log d+(3 / 2) \log N+10.17 h+\log \left(\sum_{n} D_{n}+T\right)\right) .
\end{aligned}
$$

Démonstration de l'inégalité (32). On applique la proposition 3.1, p. 15 de [Da] qui établit l'estimation suivante pour la fonction $\widetilde{\varphi}$ associée au réseau $\mathbb{Z}+\tau \mathbb{Z}:$

$$
\left|\widetilde{\varphi}(z)^{2}\right| \geq \frac{10^{-2}}{1+|\widetilde{\wp}(z)|} e^{-\frac{\pi}{2} y-4 \pi|\Im(z)|} .
$$

On utilise ensuite les estimations $|\widetilde{\wp}(1 / 2)| \leq 7.3$ et $|\widetilde{\wp}(\tau / 2)| \leq 9.7$ que l'on trouve à la page 18 et à la page 27 de [Da] respectivement (on procède de la même façon pour la fonction associée au réseau $\mathbb{Z}+\tau^{\sharp} \mathbb{Z}$ ). Comme $\varphi\left(\omega_{1} / 2\right)=\omega_{1} \widetilde{\varphi}(1 / 2)$ et $\varphi\left(\omega_{2} / 2\right)=\omega_{1} \widetilde{\varphi}(\tau / 2)$ on obtient (en appliquant le lemme 2.1)

$$
\min \left\{\left|\varphi\left(\omega_{1} / 2\right)\right|,\left|\varphi\left(\omega_{2} / 2\right)\right|\right\} \geq e^{-6.9 d h-2.5} .
$$

Des inégalités similaires sont valables pour les fonctions associées au réseau $\Lambda^{\sharp}$. L'inégalité (32) est démontrée immédiatement en revenant à la définition de $\Theta$. 
Démonstration de l'inégalité (33). Il est clair que $g\left(\omega_{1} / 2, \omega_{2} / 2\right) \in K$ (cf. lemme 3.5). La hauteur du polynôme $P$ est majorée par $(3 / 2) d^{2} h+$ $8 d^{2} \log 2+Q+\log d$ d'après le lemme 3.2. L'inégalité (24) du lemme 3.5 implique (le nombre $\Xi$ a été défini dans le lemme 3.5 ) :

$$
\begin{aligned}
h\left(g\left(\omega_{1} / 2, \omega_{2} / 2\right)\right) \leq & (3 / 2) d^{2} h+8 d^{2} \log 2+Q+\log d \\
& +\log \left(\sum_{n} D_{n}+T\right)(23.19+6 h) \\
& +T\left(\Xi+\log \left(\sum_{n} D_{n}+T\right)\right) .
\end{aligned}
$$

Nous avons supposé que $g\left(\omega_{1} / 2, \omega_{2} / 2\right) \neq 0$ et nous pouvons appliquer l'inégalité de Liouville; l'inégalité (33) est démontrée.

Démonstration de l'inégalité (34). Posons

$$
g^{\prime}=\left(\partial / \partial z_{1}\right)^{\mu_{1}}\left(\partial / \partial z_{2}\right)^{\mu_{2}} F_{P}\left(\omega_{1} / 2, \omega_{2} / 2\right) .
$$

On a, par l'hypothèse de minimalité de $\mu_{1}+\mu_{2}$ et la formule de Leibniz, que $g^{\prime}=\Theta\left(\omega_{1} / 2, \omega_{2} / 2\right) g\left(\omega_{1} / 2, \omega_{2} / 2\right)$. On utilise l'inégalité (32) pour donner une minoration de $\log \left|g^{\prime}\right|$ :

$$
\begin{aligned}
\log \left|g^{\prime}\right| \geq & \log \left|\Theta\left(\omega_{1} / 2, \omega_{2} / 2\right)\right|+\log \left|g\left(\omega_{1} / 2, \omega_{2} / 2\right)\right| \\
\geq & -(3 / 2) d^{3} h-8 d^{3} \log 2-d Q-d \log d \\
& -d \log \left(\sum_{n} D_{n}+T\right)(27+26.69 h) \\
& -d T\left(\Xi+\log \left(\sum_{n} D_{n}+T\right)\right) .
\end{aligned}
$$

Nous devons maintenant opérer un changement de base de l'espace de dérivations pour passer de la base rationnelle $\left(\partial / \partial z_{1}, \partial / \partial z_{2}\right)$ à la base $\left(\partial_{1}, \partial_{2}\right)$.

La matrice de ce changement de base est

$$
\frac{1}{\omega_{1}^{2}+\omega_{2}^{2}}\left(\begin{array}{cc}
\omega_{1} & -\omega_{2} \\
\omega_{2} & \omega_{1}
\end{array}\right)
$$

D'après le lemme 3.1, p. 287 de [Phi-Wa] on a, pour toute fonction méromorphe $A\left(z_{1}, z_{2}\right)$ de deux variables complexes,

$$
\left|\frac{\partial^{\mu_{1}}}{\partial z_{1}^{\mu_{1}}} \frac{\partial^{\mu_{2}}}{\partial z_{2}^{\mu_{2}}} A\left(z_{1}, z_{2}\right)\right| \leq\left(\frac{\left|\omega_{1}\right|+\left|\omega_{2}\right|}{\left|\omega_{1}^{2}+\omega_{2}^{2}\right|}\right)^{\mu_{1}+\mu_{2}} \max _{\mu_{1}^{\prime}+\mu_{2}^{\prime}=\mu_{1}+\mu_{2}}\left\{\left|\partial_{1}^{\mu_{1}^{\prime}} \partial_{2}^{\mu_{2}^{\prime}} A\left(z_{1}, z_{2}\right)\right|\right\} .
$$

Le lemme 2.1 implique

$$
\frac{\left|\omega_{1}\right|+\left|\omega_{2}\right|}{\left|\omega_{1}^{2}+\omega_{2}^{2}\right|} \leq \exp \{-0.35+(8 / 3) d h(E)\}
$$

en observant que $\left|1+\tau^{2}\right| \geq 7 / 4$ (nous avons supposé $\tau \in \mathfrak{F}$ ). Donc 


$$
\begin{aligned}
& \left|\frac{\partial^{\mu_{1}}}{\partial z_{1}^{\mu_{1}}} \frac{\partial^{\mu_{2}}}{\partial z_{2}^{\mu_{2}}} A\left(z_{1}, z_{2}\right)\right| \\
& \quad \leq \exp \left\{(-0.35+(8 / 3) d h(E))\left(\mu_{1}+\mu_{2}\right)\right\} \max _{\mu_{1}^{\prime}+\mu_{2}^{\prime}=\mu_{1}+\mu_{2}}\left\{\left|\partial_{1}^{\mu_{1}^{\prime}} \partial_{2}^{\mu_{2}^{\prime}} A\left(z_{1}, z_{2}\right)\right|\right\} .
\end{aligned}
$$

En appliquant l'inégalité (33) nous avons

$$
\begin{aligned}
& \log \max _{\mu_{1}^{\prime}+\mu_{2}^{\prime}=\mu_{1}+\mu_{2}}\left|\partial_{1}^{\mu_{1}^{\prime}} \partial_{2}^{\mu_{2}^{\prime}} F_{P}\left(\omega_{1} / 2, \omega_{2} / 2\right)\right| \\
& \geq-(-0.35+(8 / 3) d h(E))\left(\mu_{1}+\mu_{2}\right)+\log \left|g^{\prime}\right| \\
& \geq-(3 / 2) d^{3} h-8 d^{3} \log 2-d Q-d \log d \\
&-d \log \left(\sum_{n} D_{n}+T\right)(27+26.69 h) \\
&-d T\left(14.42+3 \log d+(3 / 2) \log N+10.17 h+\log \left(\sum_{n} D_{n}+T\right)\right) .
\end{aligned}
$$

L'inégalité (34) est démontrée et la preuve du lemme 3.8 est complète.

Lemme de Schwarz et conclusion. Nous appliquons une formule d'interpolation jouant le rôle de "principe de Schwarz approché" (lemme 2.3 de [Wa1]) ; c'est aussi ici que nous utilisons les lemmes 3.7 et 3.8. La périodicité de $f_{P}$ et la majoration (15) du lemme 3.2 impliquent que pour tout $s \in \mathbb{Z}$ et $\partial \in \mathcal{T}_{T_{0}, 2 T}$ on a

$$
\left|\partial f_{P}\left((2 s+1) \omega_{1} / 2,(2 s+1) \omega_{2} / 2\right)\right| \leq e^{-U} .
$$

Fixons un entier $0 \leq \mu_{2} \leq T$ et posons

$$
\phi(z)=\left(\partial_{2}^{\mu_{2}} F_{P}\right)\left((2 z+1) \omega_{1} / 2,(2 z+1) \omega_{2} / 2\right) .
$$

Notons $r=S+1$ et $R=8 r$, où $S$ est le paramètre défini dans (10).

Montrons que pour tout $\partial \in \mathcal{T}_{T}$ on a l'inégalité suivante :

$$
\begin{aligned}
& \max _{|z|=2(S+1)}\left\{\left|\partial F_{P}\left(\omega_{1} z / 2, \omega_{2} z / 2\right)\right|\right\} \\
& \leq e^{T \log T}\left(2\left(\frac{4 r}{R}\right)^{T_{0} S} \exp \left\{d+2 d^{2} h+Q+a(R)+b(T)\right\}\right. \\
&\left.+5\left(\frac{18 r}{S}\right)^{T_{0} S} \exp \{-U+2 T \log 2+a(S)+b(2 T)\}\right) .
\end{aligned}
$$

D'après le lemme 2.3 de [Wa1] on a l'inégalité

$$
\begin{aligned}
\max _{|z|=2 r}|\phi(z)| \leq & 2 \max _{|z|=R}|\phi(z)|\left(\frac{4 r}{R}\right)^{T_{0} S}+5\left(\frac{18 r}{S}\right)^{T_{0} S} \\
& \times \max _{\substack{0 \leq \mu_{1} \leq T_{0} \\
0 \leq s \leq S}}\left\{\left|\frac{1}{\mu_{1} !}\left(\frac{d}{d z}\right)^{\mu_{1}} \phi(s)\right|\right\} .
\end{aligned}
$$


Donnons des majorants des deux termes dans l'inégalité (37). Pour le premier terme l'on tire, d'après le lemme 3.7 inégalité (27) et par application de l'inégalité de Cauchy, $\log |\phi|_{R} \leq d+2 d^{2} h+Q+a(R)+b(T)$.

Calculons maintenant un majorant pour le deuxième terme de (37). D'après la formule de Leibniz on a

$$
\begin{aligned}
\frac{d^{\mu_{1}}}{d z^{\mu_{1}}} \phi(z)= & \sum_{i=0}^{\mu_{1}} \sum_{j=0}^{\mu_{2}}\left(\begin{array}{c}
\mu_{1} \\
i
\end{array}\right)\left(\begin{array}{c}
\mu_{2} \\
j
\end{array}\right)\left(\partial_{1}^{i} \partial_{2}^{j} \Theta\right)\left((2 z+1) \omega_{1} / 2,(2 z+1) \omega_{2} / 2\right) \\
& \times\left(\partial_{1}^{\mu_{1}-i} \partial_{2}^{\mu_{2}-j} f_{P}\right)\left((2 z+1) \omega_{1} / 2,(2 z+1) \omega_{2} / 2\right) .
\end{aligned}
$$

Comme la fonction $f_{P}$ vérifie (35) on en déduit, en utilisant l'inégalité (28), que pour tout $s=0, \ldots, S$ et pour tout $0 \leq \mu_{1} \leq T_{0}$,

$$
\begin{aligned}
\left|\left(\frac{d}{d z}\right)^{\mu_{1}} \phi(s)\right| \leq & \exp \{-U+2 T \log 2\} \\
& \times \max _{\mu_{1}^{\prime}+\mu_{2}^{\prime}=\mu_{1}+\mu_{2}|z|=S+1} \max _{1}\left|\partial_{1}^{\mu_{1}} \partial_{2}^{\mu_{2}} \Theta\left(\omega_{1} z / 2, \omega_{2} z / 2\right)\right| \\
\leq & \exp \{-U+2 T \log 2+a(S)+b(2 T)\} .
\end{aligned}
$$

En remplaçant dans (37) et en appliquant une fois de plus l'inégalité de Cauchy pour $\phi$ on termine la preuve de l'inégalité (36).

Comparaison entre estimations analytiques et estimations arithmétiques. Nous voulons montrer que $f_{P}$ a un zéro d'ordre $\geq T$ en $\left(\omega_{1} / 2, \omega_{2} / 2\right)$. Supposons le contraire. Nous en déduisons une contradiction en utilisant les estimations (34) et (36).

Supposons donc qu'il existe un monôme différentiel $\partial=\partial_{1}^{\mu_{1}} \partial_{2}^{\mu_{2}} \in \mathcal{T}_{T}$ satisfaisant $\partial f_{P}\left(\omega_{1} / 2, \omega_{2} / 2\right) \neq 0$ et choisissons $\mu_{1}, \mu_{2}$ de telle sorte que la quantité $\mu_{1}+\mu_{2} \leq T$ est minimale satisfaisant cette propriété. D'après la formule de Leibniz et le fait $\Theta\left(\omega_{1} / 2, \omega_{2} / 2\right) \neq 0$ (lemme 3.8) on a $\partial F_{P}\left(\omega_{1} / 2, \omega_{2} / 2\right)$ $\neq 0$.

Notre choix de paramètres (10) implique $U>3.59 T_{0} S$. On a donc

$$
2\left(\frac{4 r}{R}\right)^{T_{0} S} \geq 5\left(\frac{18 r}{S}\right)^{T_{0} S} e^{-U},
$$

et on déduit de l'inégalité (36) que

$$
\begin{aligned}
& \log \max _{|z|=2(S+1)}\left|\partial F_{P}\left(\omega_{1} z / 2, \omega_{2} z / 2\right)\right| \\
& \quad \leq-T_{0} S \log 2+d+2 d^{2} h+Q+a(R)+b(2 T)+\log 2+T(2 \log 2+\log T) .
\end{aligned}
$$

Notre choix de paramètres (10) implique, en utilisant le lemme 3.8, inégalité $(34)$,

$$
\begin{gathered}
T_{0} S \log 2 \leq d+2 d^{2} h+Q+a(R)+b(2 T)+\log 2 \\
+T(2 \log 2+\log T)+M_{3} .
\end{gathered}
$$


En appliquant les mêmes arguments numériques du sous-paragraphe 3.2 nous trouvons d'autre part que la quantité $T_{0} S \log 2$ est minorée par

$$
\begin{aligned}
T_{0} S \log 2 & \geq C_{3} C_{4}(\log 2) \mathcal{V} d^{3}(\log \max \{e, d\})^{3} \mathcal{L}^{3} \\
& \geq 1.4 \cdot 10^{41} \mathcal{V} d^{3}(\log \max \{e, d\})^{3} \mathcal{L}^{3},
\end{aligned}
$$

tandis que la quantité de droite $M_{4}$ satisfait la majoration

$$
\begin{aligned}
M_{4} & \leq\left(\left(2.67 C_{6}+4.1 C_{2} \log C_{1}\right) \mathcal{V}+4210.6 C_{1} C_{4}^{2}\right) d^{3}(\log \max \{e, d\})^{3} \mathcal{L}^{3} \\
& \leq\left(\left(2.67 C_{6}+4.1 C_{2} \log C_{1}\right)+17.12 C_{4}^{2} C_{1}^{-1} C_{2}\right) \mathcal{V} d^{3}(\log \max \{e, d\})^{3} \mathcal{L}^{3} \\
& \leq 1.24 \cdot 10^{41} \mathcal{V} d^{3}(\log \max \{e, d\})^{3} \mathcal{L}^{3},
\end{aligned}
$$

en appliquant la majoration dans l'encadrement (12). On obtient une contradiction avec l'inégalité (38). La preuve de la proposition 3.1 est terminée.

\section{Partie algébrique : preuve du théorème avec hauteurs naïves.}

Dans ce paragraphe nous continuons à admettre les hypothèses 2,4 jusqu'au sous-paragraphe 4.2 exclu.

Nous prouvons d'abord la majoration suivante pour $N$ (inégalité (46) plus bas) :

$$
N \leq 3.19 \cdot 10^{42} d^{2}(\log \max \{e, d\})^{2} \mathcal{L}^{2} .
$$

Les théorèmes 1 et 2 seront des conséquences de cette estimation.

Dans le sous-paragraphe 4.2 nous démontrons le théorème 1 en utilisant le lemme 10.2, p. 131 de [Da], qui est une version numériquement effective du lemme de normalisation de [Ma-Wü4], p. 19.

Dans le paragraphe 5 nous allons interpréter l'inégalité (46) en termes de hauteurs semi-stables : on aura ainsi terminé la preuve du théorème 2 .

Nous appliquons le lemme de zéros de [Den] au polynôme de la proposition 3.1 après translation du point $\exp _{G}\left(\omega_{1} / 2, \omega_{2} / 2, \omega_{1}^{\sharp} / 2, \omega_{2}^{\sharp} / 2\right)$ à l'origine de $G$ (les translations sur $G$ n'affectent pas l'ordre d'annulation le long de $A$ mais seulement la hauteur du polynôme $P$ ). Le lemme de zéros de [Den] améliore le résultat principal de [Phi2] en supprimant le degré des formules d'addition dans l'estimation finale $\left({ }^{7}\right)$.

Pour une sous-variété abélienne $\mathfrak{H}$ de $G$ notons $\varrho$ la codimension de $A \cap \mathfrak{H}$ dans $A$ et $r$ la codimension de $\mathfrak{H}$ dans $G$. Nous reproduisons ici l'énoncé du lemme de zéros (théorème 1 ) de [Den] dans la version qui nous intéresse : on note $\mathfrak{R}=\mathbb{C}\left[\underline{X}_{n}\right]_{1 \leq n \leq 4}$ où $\underline{X}_{n}=\left(X_{0, n}, X_{1, n}, X_{2, n}\right)$.

$\left({ }^{7}\right)$ Observons que le lemme de zéros de [Phi1] améliore le résultat de [Phi2] du côté des multiplicités d'annulation. Le lemme de zéros de [Den] permet de gagner un facteur $2^{4}$ sur le résultat final, par rapport au résultat principal de [Phi2] (4 est la dimension de $G$ ) et le lemme de zéros de [Phi1] permet de gagner essentiellement un facteur 2! (2 est la dimension de l'espace de dérivations, d'exponentielle $A$ ). Il est fort probable que ces deux améliorations soient en fait compatibles en caractéristique nulle, mais nous choisirons le résultat de [Den] à défaut — pour l'instant — d'une référence adéquate. 
Proposition 4.1 (Lemme de multiplicités). Fixons $D_{1}, \ldots, D_{4}, T$ des entiers positifs, soit $P$ un polynôme de $\mathfrak{R}$ multi-homogène de multidegré $\leq \underline{D}$. Supposons que P s'annule en $\exp _{G}\left(\omega_{1} / 2, \omega_{2} / 2, \omega_{1}^{\sharp} / 2, \omega_{2}^{\sharp} / 2\right)$ le long de $A$ à un ordre supérieur à $T$ et qu'il ne soit pas identiquement nul sur $G$. Il existe alors une sous-variété abélienne $\mathfrak{H}$ de $G$, différente de $G$, telle que pour tout multi-indice $\underline{\alpha}$ satisfaisant $0 \leq \alpha_{i} \leq 1$ et $|\underline{\alpha}|=\operatorname{dim}(\mathfrak{H})$ on ait

$$
\left(\begin{array}{c}
{[(T-1) / 4]+\varrho} \\
\varrho
\end{array}\right) \operatorname{dim}(\mathfrak{H}) ! \operatorname{deg}_{\underline{\alpha}}(\mathfrak{H}) \leq 3^{4} \cdot 4 ! \prod_{n=1}^{4} D_{n}^{1-\alpha_{n}} .
$$

4.1. Cas des isogénies normalisées. Nous avons démontré la proposition 3.1 ; il existe donc un polynôme $P$ de $\mathfrak{R}$ satisfaisant les hypothèses de la proposition 4.1. Appliquons cette proposition : il existe alors une sous-variété abélienne $\mathfrak{H}$ de $G$, non égale à $G$, dont les degrés partiels $\operatorname{deg}_{\underline{\alpha}}(\mathfrak{H})$ satisfont l'inégalité (39).

D'après le lemme 3.1 et le choix de paramètres (10) on a $\mathfrak{H} \in \mathfrak{A}$ (on utilise l'identité (11)). L'ensemble $\mathfrak{A}$ a été défini dans le sous-paragraphe 3.1.

Si $\mathfrak{H}=A$ ou $A \subset \mathfrak{H}$ est de codimension 1 alors $\mathfrak{H}$ est non déployée. Si $\mathfrak{H} \subset A$ est de codimension 1 alors $\mathfrak{H}$ est déployée si et seulement si $m_{1,2}=0$, cas dégénéré que nous avons exclu (cf. hypothèse 3 du sous-paragraphe 2.2). Ainsi, grâce à l'hypothèse $3, \mathfrak{H}$ est non déployée.

À l'exception de cinq sous-variétés abéliennes notées $A, A_{1}, A_{2}, B_{1}, B_{2}$ que nous traiterons différemment (dans le lemme 4.1), nous montrons que toute sous-variété abélienne $\mathfrak{H}$ donnée par le lemme de multiplicités a deux multidegrés non nuls correspondant à des indices distincts $\operatorname{deg}_{\underline{\alpha}}(\mathfrak{H})$ et $\operatorname{deg}_{\alpha^{\prime}}(\mathfrak{H})$ dont le produit est un polynôme de degré $\leq 2$ en $\log N$ et $h$ : ce produit majore $N$ en utilisant le lemme d'isogénie 2.2.

Pour traiter les sous-variétés abéliennes $A, A_{1}, A_{2}, B_{1}, B_{2}$ nous utiliserons le sous-paragraphe qui suit.

Cardinaux de groupes cycliques finis. Nous rappelons les sous-espaces vectoriels $Z_{1}, Z_{2}, Z$ de $T_{\mathbb{C}}$ définis dans (8). Notons $F_{1}$ l'intersection de $Z$ avec l'hyperplan d'équation $z_{1}=0$ dans $\mathbb{C}^{4}$ et $F_{2}$ l'intersection de $Z$ avec l'hyperplan $z_{2}=0$. Posons $A_{1}=\exp _{G}\left(Z_{1}\right)$ et $A_{2}=\exp _{G}\left(Z_{2}\right)$. Notons aussi $B_{1}=\exp _{G}\left(F_{1}\right)$ et $B_{2}=\exp _{G}\left(F_{2}\right)$. Nous remarquons que $A$ est la composante neutre de $A_{1} \cap A_{2}$.

Lemme 4.1. Notons $(\cdot, \cdot)$ le plus grand commun diviseur entre deux entiers rationnels et notons $\sigma=\left(m_{1,2}, m_{2,2}\right)$ et $\delta=\left(m_{1,1}, m_{1,2}\right)$. Dans l'hypothèse où l'isogénie normalisée $\phi$ définie par les équations (7) a son noyau cyclique, on a les égalités suivantes:

$$
\operatorname{Card}\left(A \cap\left(E \times E \times 0^{\sharp} \times 0^{\sharp}\right)\right)=\operatorname{Card}\left(A \cap\left(0 \times 0 \times E^{\sharp} \times E^{\sharp}\right)\right)=N,
$$




$$
\begin{aligned}
& \operatorname{Card}\left(B_{1} \cap\left(0 \times 0 \times E^{\sharp} \times E^{\sharp}\right)\right)=\operatorname{Card}\left(A_{2} \cap\left(E \times 0 \times 0^{\sharp} \times 0^{\sharp}\right)\right)=N / \sigma, \\
& \operatorname{Card}\left(B_{2} \cap\left(E \times E \times 0^{\sharp} \times 0^{\sharp}\right)\right)=\operatorname{Card}\left(A_{1} \cap\left(0 \times 0 \times E^{\sharp} \times 0^{\sharp}\right)\right)=N / \delta .
\end{aligned}
$$

Démonstration. Les égalités (40) sont prouvées dans le lemme p. 15 de [Ma-Wü4]. Démontrons les égalités (41). Le sous-espace vectoriel $F_{1}$ est défini par

$$
\left\{\begin{array}{l}
z_{1}=0, \\
z_{1}^{\sharp}=m_{1,2} z_{2}, \\
z_{2}^{\sharp}=m_{2,2} z_{2} .
\end{array}\right.
$$

Le cardinal $l=\operatorname{Card}\left(B_{1} \cap\left(0 \times 0 \times E^{\sharp} \times E^{\sharp}\right)\right)$ est le nombre de solutions $\left(z_{1}^{\sharp}, z_{2}^{\sharp}\right)$ de (43) modulo $\Lambda^{\sharp 2}$ pour $z_{2} \in \Lambda$. L'isogénie $\phi$ est cyclique : la deuxième équation de (43) admet $N /\left(m_{1,2}, N\right)$ solutions modulo $\Lambda^{\sharp}$ et la troisième $N /\left(m_{2,2}, N\right)$. On a donc $l=N /\left(m_{1,2}, m_{2,2}\right)$.

Le cardinal $l=\operatorname{Card}\left(A_{2} \cap\left(E_{1} \times 0 \times 0^{\sharp} \times 0^{\sharp}\right)\right)$ est le nombre de $z_{1} \in \mathbb{C}$ distincts modulo $\Lambda$ tels que $z_{1}=m_{1,2} \alpha-m_{2,2} \beta$, pour $\alpha, \beta \in \Lambda^{\sharp} / N$; le fait que l'isogénie $\phi$ est cyclique permet de conclure la preuve des deux égalités (41).

Les égalités (42) se démontrent de manière tout à fait analogue. Le sousespace linéaire $F_{2}$ s'identifie à l'intersection des trois hyperplans $z_{1}^{\sharp}=0$, $N z_{1}=-m_{1,2} z_{2}^{\sharp}, N z_{2}=m_{1,1} z_{2}$, et le cardinal $l=\operatorname{Card}\left(B_{2} \cap\left(E \times E \times 0^{\sharp} \times 0^{\sharp}\right)\right)$ est égal à $N /\left(m_{1,1}, m_{1,2}\right)$, toujours en utilisant le fait que l'isogénie $\phi$ est cyclique. Mêmes raisonnements pour la deuxième identité ; la démonstration du lemme 4.1 est complète.

Estimation des quantités $\sigma$ et $\delta$

Lemme 4.2. On a les deux inégalités suivantes concernant les nombres $\sigma, \delta$ définis dans le lemme 4.1 :

$$
y \sigma, y^{\sharp} \delta \leq d(117 \log N+348 d h(E)) .
$$

Démonstration. Soit $\Lambda^{(\sigma)}$ le réseau de $\mathbb{C}$ engendré par $\omega_{1}$ et $\sigma \omega_{2}$. Soit $E^{(\sigma)}$ la courbe elliptique $\mathbb{C} / \Lambda^{(\sigma)}$. Nous avons $\Lambda^{(\sigma)} \subset \Lambda$ et il existe une isogénie normalisée $\psi_{\sigma}: E^{(\sigma)} \rightarrow E$ (représentée par la matrice $\left(\begin{array}{ll}1 & 0 \\ 0 & \sigma\end{array}\right)$ ). Il existe de même une isogénie normalisée $E^{\sharp} \rightarrow E^{(\sigma)}$ qui factorise $\phi$ par $\psi_{\sigma}$. Comme $\Lambda^{\sharp} \subset \Lambda^{(\sigma)} \subset \Lambda$ on a que $E^{(\sigma)}$ peut être définie sur $K$. On a

$$
y \sigma=y^{(\sigma)} \leq \frac{3}{2} \max \left\{1, d h\left(j_{E^{(\sigma)}}\right)\right\} \leq \frac{3}{2} d(78 \log \sigma+232 d h(E)) .
$$

On trouve la première inégalité d'après le lemme 2.1(i), la deuxième inégalité en observant que $E^{(\sigma)}$ s'identifie à un quotient de $E$ par un sous-groupe cyclique de cardinal $\sigma$; on applique alors le lemme 10.2, p. 131 de [Da]. L'estimation (44) pour la quantité $y \sigma$ découle aisément.

Pour démontrer l'estimation pour la quantité $y^{\sharp} \delta$ nous remarquons que $\Lambda^{\sharp} \subset \Lambda^{(\delta)} \subset \Lambda, \Lambda^{(\delta)}$ étant le réseau de $\mathbb{C}$ engendré par $\omega_{1}^{\sharp} / \delta$ et $\omega_{2}^{\sharp}$, par 
conséquent $E^{(\delta)} \cong \mathbb{C} / \Lambda^{(\delta)}$ est définie sur $K$; la suite de la démonstration est analogue au pas ci-dessus. On termine ainsi la preuve du lemme 4.2.

Analyse de sous-variétés abéliennes de $G$

LEMME 4.3. Soit $\mathfrak{H} \subset G=E^{2} \times E^{\sharp 2}$ une sous-variété abélienne non déployée de dimension $b=1$ ou $b=3$ et supposons que $\alpha=\operatorname{deg}_{(1,0,0,0)}(\mathfrak{H}) \times$ $\operatorname{deg}_{(0,0,1,0)}(\mathfrak{H}) \neq 0$ si $b=1$ ou $\alpha=\operatorname{deg}_{(1,1,1,0)}(\mathfrak{H}) \operatorname{deg}_{(0,1,1,1)}(\mathfrak{H}) \neq 0$ si $b=3$. Supposons de plus, si $\operatorname{dim} \mathfrak{H}=3$, que $\mathfrak{H}$ contienne A. Alors il existe une isogénie $\psi$ liant $E$ et $E^{\sharp}$ dont le degré satisfait $\operatorname{deg} \psi \leq 3^{6} \alpha$.

Démonstration. Supposons d'abord que $\mathfrak{H}$ ait pour dimension 1 et considérons la projection $\pi: G \rightarrow E \times E^{\sharp}$ définie par $\pi\left(x_{1}, x_{2}, x_{3}, x_{4}\right)=\left(x_{1}, x_{3}\right)$. L'hypothèse sur la non nullité des multi-degrés implique que $\pi$ induit une isogénie (soit $n$ son degré) de $\mathfrak{H}$ sur son image $\mathfrak{H}^{\prime}$ dans $E \times E^{\sharp}$ et que cette image est non déployée. On a $0 \neq n^{2} \operatorname{deg}_{(1,0)}\left(\mathfrak{H}^{\prime}\right) \operatorname{deg}_{(0,1)}\left(\mathfrak{H}^{\prime}\right) \leq \alpha$. Le lemme 2.2 appliqué à $\mathfrak{H}^{\prime}$ implique alors qu'il existe une isogénie liant $E$ et $E^{\sharp}$ dont le degré $N$ satisfait $N \leq 3^{2} \alpha$ (en tenant compte du degré du plongement de Weierstrass et en utilisant le théorème de Bézout).

Nous pouvons traiter le cas où $\mathfrak{H}$ a pour dimension 3. Dans ce cas on a $\mathfrak{H} \supset A$. Observons que le sous-groupe algébrique $\mathfrak{H}^{\prime}=\mathfrak{H} \cap\left(0 \times E \times 0^{\sharp} \times E^{\sharp}\right)$ de $\mathfrak{H}$ ne peut pas être fini (le sous-groupe algébrique de $G$ engendré par $\mathfrak{H}$ et $0 \times E \times 0^{\sharp} \times E^{\sharp}$ a pour dimension au plus 4); il a donc pour dimension 1 ou 2. Si $\operatorname{dim} \mathfrak{H}^{\prime}=2$ alors $A \cap\left(0 \times E \times 0^{\sharp} \times E^{\sharp}\right)$ a pour dimension 1 et on est dans le cas dégénéré, que nous avons exclu. On projette $\mathfrak{H}^{\prime}$ dans $E \times E^{\sharp}$; l'image est un sous-groupe algébrique non déployé que nous notons toujours $\mathfrak{H}^{\prime}$. D'après le lemme 2.2 il existe une isogénie liant $E$ et $E^{\sharp}$ dont le degré $N$ satisfait $N \leq 3^{2} \operatorname{deg}_{(1,0)}\left(\mathfrak{H}^{\prime}\right) \operatorname{deg}_{(0,1)}\left(\mathfrak{H}^{\prime}\right) \leq 3^{6} \alpha$. Le lemme est démontré.

D'après le lemme de multiplicités (proposition 4.1), il existe une sousvariété abélienne $\mathfrak{H} \subsetneq G$ telle que les inégalités (39) soient satisfaites. Notre choix de paramètres (10) et le lemme 3.1 impliquent que cette sous-variété abélienne satisfait une des conditions suivantes : $0 \neq \mathfrak{H} \subsetneq A, \mathfrak{H}=A, A \subsetneq \mathfrak{H}$.

Nous allons déduire de chaque cas des estimations pour des produits de multi-degrés non nuls, et ensuite des estimations pour $N$.

CAS $\mathfrak{H} \subsetneq A$. La sous-variété abélienne $\mathfrak{H}$ est non déployée. Si elle était déployée on aurait $\mathfrak{H}=0 \times 0 \times \mathfrak{H}_{1}$ ou $\mathfrak{H}=\mathfrak{H}_{2} \times 0^{\sharp} \times 0^{\sharp}$, ce qui entraînerait par (40) que $\mathfrak{H}$ est finie, donc égale à 0 par connexité; ce cas est exclu.

Soit $\pi$ la projection utilisée dans la démonstration du lemme 4.2. Soit $\pi(\mathfrak{H}) \subset E \times E^{\sharp}$ l'image de $\mathfrak{H}$ par $\pi$. Si $\pi(\mathfrak{H})$ est non déployée alors

$$
\operatorname{deg}_{(1,0,0,0)}(\mathfrak{H}) \operatorname{deg}_{(0,0,1,0)}(\mathfrak{H}) \neq 0
$$

et 
$\operatorname{deg}_{(1,0,0,0)}(\mathfrak{H}) \operatorname{deg}_{(0,0,1,0)}(\mathfrak{H})$

$$
\begin{aligned}
& \leq 3^{8} \cdot(4 !)^{2} \cdot 4^{2} T^{-2}\left(\prod_{n} D_{n}\right) D_{2} D_{4} \\
& \leq 3^{8} \cdot(4 !)^{2} \cdot 4^{2} \cdot 1.66 \cdot 10^{31} \mathcal{V}^{-2} d^{2}(\log \max \{e, d\})^{2} \frac{\mathcal{L}^{2}}{y y^{\sharp}} \\
& \leq 10^{39} d^{2}(\log \max \{e, d\})^{2} \mathcal{L}^{2} .
\end{aligned}
$$

D'après le lemme 4.3 on obtient dans ce cas

$$
N \leq 7.29 \cdot 10^{41} d^{2}(\log \max \{e, d\})^{2} \mathcal{L}^{2} .
$$

Si $\pi(\mathfrak{H})$ est déployé, il faut distinguer trois cas selon que $\pi(\mathfrak{H})$ est égal à l'une des sous-variétés abéliennes $0 \times 0^{\sharp}, E \times 0^{\sharp}$ ou $0 \times E^{\sharp}$.

(a) Si $\pi(\mathfrak{H})=0 \times 0^{\sharp}$ alors $\mathfrak{H} \subset\left(0 \times E \times 0^{\sharp} \times E^{\sharp}\right)$. Comme $\mathfrak{H} \subset A$ on a $\operatorname{dim}\left(A \cap\left(0 \times E \times 0^{\sharp} \times E^{\sharp}\right)\right)=1$ et on est dans le cas dégénéré, que nous avons exclu.

(b) Si $\pi(\mathfrak{H})=0 \times E^{\sharp}$ alors $\mathfrak{H}=B_{1}$ et le multi-degré $\operatorname{deg}_{(0,1,0,0)}\left(B_{1}\right)$ est non nul. On trouve

$$
\operatorname{deg}_{(0,1,0,0)}\left(B_{1}\right) \leq 3^{4} \cdot 4 ! \cdot 4 D_{1} D_{3} D_{4} T^{-1} \leq 3.17 \cdot 10^{19} d \log \max \{e, d\} \mathcal{L} y .
$$

D'après les identités du lemme 4.1 et les inégalités du lemme 4.2 on tire dans ce cas

$$
\begin{aligned}
N & \leq 3 \operatorname{deg}_{(0,1,0,0)}\left(B_{1}\right) \sigma \\
& \leq 9.49 \cdot 10^{19} d \log \max \{e, d\} \mathcal{L} y \sigma \\
& \leq 9.49 \cdot 10^{19} d^{2} \log \max \{e, d\} \mathcal{L}(117 \log N+384 d h(E)) .
\end{aligned}
$$

(c) Si $\pi(\mathfrak{H})=E \times 0^{\sharp}$ alors $\mathfrak{H}=B_{2}$ et le multi-degré $\operatorname{deg}_{(0,0,0,1)}\left(B_{2}\right)$ est non nul; on a $\operatorname{deg}_{(0,0,0,1)}\left(B_{2}\right) \leq 3.17 \cdot 10^{19} d \log \max \{e, d\} \mathcal{L} y^{\sharp}$. Les lemmes 4.1 et 4.2 impliquent une fois de plus l'estimation (45).

CAS $\mathfrak{H}=A$. Dans ce cas le produit $\operatorname{deg}_{(1,1,0,0)}(A) \operatorname{deg}_{(0,0,1,1)}(A)$ est non nul et on trouve

$$
\begin{aligned}
\max \left\{\operatorname{deg}_{(1,1,0,0)}(A), \operatorname{deg}_{(0,0,1,1)}(A)\right\} & \leq 3^{4} \cdot 4 ! \cdot 2^{-1} \max \left\{D_{3} D_{4}, D_{1} D_{2}\right\} \\
& \leq 9.72 \cdot 10^{38} d^{2}(\log \max \{e, d\})^{2} \mathcal{L}^{2} .
\end{aligned}
$$

La dernière quantité constitue aussi une majoration pour $N$, en utilisant l'identité (40) du lemme 4.1.

CAS $A \subset \mathfrak{H}$. On a $\mathfrak{H}=\exp _{G}(W)$ où $W$ est un sous-espace linéaire de $T_{\mathbb{C}} \cong \mathbb{C}^{4}$ défini par une équation $\lambda_{1}\left(m_{1,1} z_{1}+m_{1,2} z_{2}-z_{1}^{\sharp}\right)+\lambda_{2}\left(m_{2,1} z_{1}+\right.$ $\left.m_{2,2} z_{2}-z_{2}^{\sharp}\right)=0$, où $\lambda_{1}$ et $\lambda_{2}$ sont deux nombres complexes non tous les deux nuls. Comme $\mathfrak{H} \supset A, \mathfrak{H}$ est non déployée. 
Supposons que la composante neutre $\mathfrak{H}^{\prime}$ de $\left(\mathfrak{H} \cap\left(0 \times E \times 0^{\sharp} \times E^{\sharp}\right)\right)$ soit non déployée : le produit $\operatorname{deg}_{(1,1,1,0)}(\mathfrak{H}) \operatorname{deg}_{(1,0,1,1)}(\mathfrak{H})$ est non nul et satisfait

$$
\begin{aligned}
\operatorname{deg}_{(1,1,1,0)}(\mathfrak{H}) \operatorname{deg}_{(1,0,1,1)}(\mathfrak{H}) & \leq 3^{8} \cdot(3 !)^{-2} \cdot(4 !)^{2} D_{2} D_{4} \\
& \leq 4.38 \cdot 10^{39} \cdot d^{2}(\log \max \{e, d\})^{2} \mathcal{L}^{2} .
\end{aligned}
$$

En appliquant le lemme 4.3 on déduit la majoration

$$
N \leq 3.19 \cdot 10^{42} d^{2}(\log \max \{e, d\})^{2} \mathcal{L}^{2} .
$$

Puisque $m_{1,2} \neq 0$, si $\mathfrak{H}^{\prime}$ est déployée on a deux cas suivant que $\mathfrak{H}=A_{1}$ ou $\mathfrak{H}=A_{2}$. Dans le premier cas on a $\lambda_{2}=0$ et $\operatorname{deg}_{(1,1,0,1)}\left(A_{1}\right) \neq 0$. Donc

$$
\operatorname{deg}_{(1,1,0,1)}\left(A_{1}\right) \leq 3^{4} \cdot 4 ! \cdot(3 !)^{-1} D_{3} \leq 3.24 \cdot 10^{20} d \log \max \{e, d\} \mathcal{L} y^{\sharp} .
$$

Les lemmes 4.1 et 4.2 impliquent la majoration

$$
N \leq 9.72 \cdot 10^{20} d^{2} \log \max \{e, d\} \mathcal{L}(117 \log N+384 d h(E)) .
$$

Dans le deuxième cas on a $\lambda_{1} m_{1,2}+\lambda_{2} m_{2,2}=0$; dans ce cas le multi-degré $\operatorname{deg}_{(0,1,1,1)}\left(A_{2}\right)$ est non nul et on a

$$
\operatorname{deg}_{(0,1,1,1)}\left(A_{2}\right) \leq 3^{4} \cdot 4 ! \cdot(3 !)^{-1} D_{1} \leq 3.24 \cdot 10^{20} d \log \max \{e, d\} \mathcal{L} y .
$$

On applique les lemmes 4.1 et 4.2 et on parvient à la même majoration de $N$ que dans le cas précédent.

L'analyse des sous-variétés abéliennes de $G$ est terminée. Observons que l'estimation pour $N$ la plus défavorable est celle qui est obtenue pour $\operatorname{dim} \mathfrak{H}=3$ en "position générale", c'est-à-dire l'estimation (46); celle-ci est valable inconditionnellement pour tout $\mathfrak{H}$ comme dans le lemme de multiplicité 4.1. Nous oublions les autres estimations obtenues et nous continuons nos calculs avec celle-ci.

4.2. Du cas normalisé au cas général. Nous éliminons les hypothèses 2 , 4 , et nous démontrons le théorème 1 . Soient $E$ et $E_{1}^{\sharp}$ deux courbes elliptiques définies sur un corps de nombres $K$ et supposons donnée une isogénie $\phi_{1}$ : $E \rightarrow E_{1}^{\sharp}$ dont le noyau cyclique a cardinal $N$.

Nous avons supposé dans tout notre texte que $E$ est sans multiplication complexe; l'isogénie $\phi_{1}$ est donc définie sur $K$.

Supposons dans un premier temps que tous les points de 2-torsion de $E$ et $E_{1}^{\sharp}$ soient définis sur $K$. Notons $E^{\sharp}=E / \Phi$; c'est une courbe elliptique ayant un modèle de Weierstrass comme dans (1) défini sur $K$, isogène à $E$ et $K$-isomorphe à $E_{1}^{\sharp}$. De plus l'isogénie induite $E \rightarrow E^{\sharp}$ est normalisée. Nous considérons en fait sa duale, $\phi: E^{\sharp} \rightarrow E$, qui est elle aussi normalisée. Les hypothèses 2 et 4 sont donc satisfaites et nous pouvons appliquer tous les arguments développés dans les paragraphes précédents.

Nous obtenons la majoration (46) pour $N$, qui dépend des hauteurs $h(E)$ et $h\left(E^{\sharp}\right)$ ainsi que de $\log N$. Le premier pas est d'éliminer la dépendance en 
$h\left(E^{\sharp}\right)$ : nous appliquons le lemme 10.2, p. 131 de [Da] $\left(^{8}\right)$. Plus précisément nous utilisons les inégalités

$$
\begin{aligned}
h\left(1: g_{2}^{\sharp}: g_{3}^{\sharp}\right) & \leq 13 \log N+9 d h(E)+6 d \log 4+19.31, \\
h\left(j_{E^{\sharp}}\right) & \leq 78 \log N+232 d h(E) .
\end{aligned}
$$

On obtient

$$
\mathcal{L} \leq 241 d h(E)+92 \log N+6 d \log 4+19.31
$$

En appliquant cette inégalité à l'estimation (46) nous trouvons

$$
\begin{aligned}
N \leq \operatorname{deg} \psi \leq & 2.94 \cdot 10^{44} d^{2}(\log \max \{e, d\})^{2} \\
& \times(0.3+0.1 d+2.7 d h(E)+\log N)^{2} .
\end{aligned}
$$

Le deuxième pas est d'éliminer la dépendance en $\log N$ introduite par la méthode de Baker; nous utilisons le lemme suivant.

Lemme 4.4. Soient u, a deux nombres réels supérieurs ou égaux à e, soit $N$ un entier rationnel $\geq 3$ et supposons que $N \leq u(a+\log N)^{2}$. On a alors

$$
N \leq \max \left\{5504,4 u a^{2}, u(a+2 \log u+4 \log 2)^{2}\right\} .
$$

Démonstration. Il suffit d'appliquer les propriétés de convexité de la fonction $\log$.

On applique l'estimation (48) tenant compte de l'inégalité (46). On pose $u=2.94 \cdot 10^{44} d^{2}(\log \max \{e, d\})^{2}, a=0.3+0.1 d+2.7 d h(E)$. En remarquant que $d \geq 2$ et que nous avons $h(E) \geq 2$ on a

$$
\begin{aligned}
N & \leq 4 u(a+2 \log u+4 \log 2)^{2} \\
& \leq 9.4 \cdot 10^{45} d^{4}(\log \max \{e, d\})^{2} h(E)^{2} .
\end{aligned}
$$

Observons que si $K$ ne contient pas tous les nombres algébriques $\wp\left(\omega_{1} / 2\right)$, $\wp\left(\omega_{2} / 2\right), \wp^{\sharp}\left(\omega_{1}^{\sharp} / 2\right), \wp^{\sharp}\left(\omega_{2}^{\sharp} / 2\right)$, il existe une extension de degré au plus $3^{4}$ de $K$ qui contient tous ces nombres. Donc

$$
N \leq 10^{54} d^{4} \log (81 d)^{2} h(E)^{2},
$$

le théorème 1 est démontré.

\section{Majoration de degrés d'isogénie avec hauteurs semi-stables.}

Le résultat principal de ce paragraphe est le lemme 5.2, qui n'est qu'une version numériquement effective de la proposition 2.1 de [Sil], p. 256.

Notons $j(\tau)$ la fonction modulaire elliptique $j(\tau)=1 / q_{\tau}+744$ $+\sum_{n>0} c_{n} q_{\tau}^{n}$ où l'on a posé $q_{\tau}=\exp \{2 \pi i \tau\}$. Démontrons préalablement les inégalités suivantes:

$\left({ }^{8}\right)$ Notons que ce lemme de normalisation est une version "hauteurs naïves" de l'inégalité $h_{\mathcal{F}}\left(E^{\sharp}\right) \leq h_{\mathcal{F}}(E)+(1 / 2) \log N$ de [Fal]. Ce lemme 10.2 de [Da] rajoute un facteur de proportionnalité $d^{2}$ dans l'estimation finale (2), par rapport à l'inégalité de Faltings ci-dessus mentionnée; cf. inégalité (47). Pour cela nous aurons un facteur $d^{4}$ dans l'estimation 1 au lieu d'un facteur $d^{2}$ plus naturel. 
Lemme 5.1. Soit $\tau \in \mathfrak{F}, \tau=x+i y$. On a

(i) $|\log \max \{1,|j(\tau)|\}-2 \pi y| \leq 8.36$,

(ii) $|\log \log \max \{e,|j(\tau)|\}-\log y| \leq 2.77$.

Démonstration. (i) Prouvons d'abord $\log \max \{1,|j(\tau)|\}-2 \pi y \leq 8.36$. D'après [He], p. 189, Satz 2c), on a $|j(\tau)| \leq 3\left(e^{2 \pi y}+e^{2 \pi / y}\right)$. Il suit des propriétés de la fonction $e^{2 \pi y}+e^{2 \pi / y}$ et de l'inégalité $y \geq \sqrt{3} / 2$ que

$$
\log \max \{1,|j(\tau)|\} \leq \log 3+\log \left(e^{2 \pi y}+1416\right) \leq 8.36+2 \pi y .
$$

Maintenant montrons que $\log \max \{1,|j(\tau)|\}-2 \pi y \geq-7.09$. D'après [Fa-Ph], p. 187, lemme 1(iii), si $|j(\tau)| \geq 2$ on a bien l'inégalité que l'on cherche. Si $|j(\tau)|<2$ on utilise l'inégalité (6) pour prouver complètement la première inégalité (i).

(ii) Observons d'abord $\log \log \max \{e,|j(\tau)|\}-\log y \geq-0.41$ : c'est une application directe de l'inégalité (6).

Vérifions maintenant $\log \log \max \{e,|j(\tau)|\}-\log y \leq 2.77$. Il est clair que (i) est valable en remplaçant $\log \max \{1,|j(\tau)|\} \operatorname{par} \log \max \{e,|j(\tau)|\}$, puisque la plus petite valeur prise par la fonction $e^{2 \pi y}+e^{2 \pi / y}$ dans l'intervalle $(\sqrt{3} / 2,+\infty)$ est $6 e^{2 \pi}$ et $\log \left(6 e^{2 \pi}\right) \approx 8.07>e$. Comme $y \geq \sqrt{3} / 2$, on termine la preuve de (ii) par convexité de la fonction log.

Pour tout $v \in M_{K}^{\infty}$ soit $\tau_{v} \in \mathfrak{F}$ tel que $E\left(\bar{K}_{v}\right) \cong \mathbb{C} /\left(\mathbb{Z}+\tau_{v} \mathbb{Z}\right), \bar{K}_{v}$ désignant une clôture algébrique du complété de $K$ par rapport à $v$.

Lemme 5.2. Pour toute courbe elliptique $E$ définie sur un corps de nombres $K$ on a

$$
h\left(j_{E}\right) \leq 94.3+24 \max \left\{1, h_{\mathcal{F}}(E)\right\} .
$$

Démonstration. L'inégalité (50) est une conséquence directe de l'inégalité suivante :

$$
\left|h\left(j_{E}\right)-12 h_{\mathcal{F}}(E)\right| \leq 6 \log \left(1+h\left(j_{E}\right)\right)+47.15 .
$$

Pour le voir, observons que si $x$ est réel tel que $x>48$, alors $x / 2-$ $6 \log (1+x)>0$; il suffit maintenant de poser $x=h\left(j_{E}\right)$ et d'utiliser l'inégalité (51).

Pour prouver l'inégalité (51) il suffit d'expliciter les constantes dans la proposition 2.1 de [Sil], p. 256. Nous détaillons ici la démonstration.

Notons $\Delta_{\left.E\right|_{K}}$ le discriminant absolu de $E$ sur $K$ et $\Delta(\tau)$ la fonction $(2 \pi)^{-12} q_{\tau} \prod_{n=1}^{\infty}\left(1-q_{\tau}^{n}\right)^{24}$. On applique la formule de la proposition 1.1, p. 254 de [Sil], qui est valable pour toute extension $K^{\prime}$ de $K$ de degré $d^{\prime}$ $\operatorname{sur} \mathbb{Q}$ :

$$
12 d^{\prime} h\left(\left.E\right|_{K^{\prime}}\right)=\log \left|N_{K^{\prime} \mid \mathbb{Q}} \Delta_{\left.E\right|_{K^{\prime}}}\right|-\sum_{v \in M_{K^{\prime}}^{\infty}} n_{v} \log \left(\left|\Delta\left(\tau_{v}\right)\right| y_{v}^{6}\right) .
$$


L'exercice de [Sil], p. 256 implique, pour toute place archimédienne $v$ d'une extension finie $K^{\prime}$ de $K$,

$$
\left|\log \left(\left|\Delta\left(\tau_{v}\right)\right| \Im(\tau)^{6}\right)+2 \pi \Im\left(\tau_{v}\right)+12 \log 2 \pi-6 \log \Im\left(\tau_{v}\right)\right| \leq 1 / 9 .
$$

Nous utilisons les inégalités (i) et (ii) du lemme 5.1 :

$$
\begin{aligned}
& \left|\log \left(\left|\Delta\left(\tau_{v}\right)\right| \Im\left(\tau_{v}\right)^{6}\right)+\log \max \left\{1,\left|j\left(\tau_{v}\right)\right|\right\}\right| \\
& \quad \leq 1 / 9+7.24+12 \log 2 \pi+6 \cdot 2.77+6 \log \log \max \left\{e,\left|j\left(\tau_{v}\right)\right|\right\} .
\end{aligned}
$$

Si $K^{\prime}$ est une extension de $K$ telle que $E$ est semi-stable sur $K^{\prime}$ alors

$$
d^{\prime} h\left(j_{E}\right)=\log \left|N_{K^{\prime} \mid \mathbb{Q}} \Delta_{\left.E\right|_{K^{\prime}}}\right|+\sum_{v \in M_{K^{\prime}}^{\infty}} n_{v} \log \max \left\{1,\left|j\left(\tau_{v}\right)\right|\right\}
$$

(voir p. 257 de [Sil]). Observons que

$$
\begin{aligned}
\sum_{v \in M_{K^{\prime}}^{\infty}} n_{v} \log \log \max \left\{e,\left|j\left(\tau_{v}\right)\right|\right\} & \leq d^{\prime} \log \left(1+\frac{1}{d} \sum_{v \in M_{K^{\prime}}^{\infty}} \log \max \left\{\left|j_{E}\right|_{v}, 1\right\}\right) \\
& \leq d \log \left(1+h\left(j_{E}\right)\right) .
\end{aligned}
$$

On somme (53) sur toutes les places archimédiennes $v$ de $K^{\prime}$ avec des poids correspondant aux degrés locaux $n_{v}$, tenant compte de l'inégalité (54), et on termine en utilisant l'inégalité (54) et l'identité (52).

Lemme 5.3 (Normalisation des modèles de Weierstrass). Soit $E$ une courbe elliptique définie sur un corps de nombres $K$ sans multiplication complexe avec un modèle de Weierstrass $y^{2}=4 x^{3}-g_{2} x-g_{3}$. On a les propriétés suivantes.

- Il existe une extension $K^{\prime}$ de $K$ de degré relatif 2 telle que $E$ admette sur $K^{\prime}$ un modèle de Weierstrass

$$
y^{2}=4 x^{3}-2 x-g_{3}^{\prime} .
$$

- La hauteur de ce modèle (55) vérifie

$$
h\left(1: 2: g_{3}^{\prime}\right) \leq h\left(g_{3}^{\prime}\right)+\log 2 \leq 8.5+(1 / 2) h\left(j_{E}\right) .
$$

Démonstration. Le fait de supposer qu'il n'y ait pas de multiplication complexe nous permet de supposer $g_{2} \neq 0$. Le modèle de Weierstrass de $E$,

$$
y^{2}=4 x^{3}-2 x-\left(\frac{8}{27}\left(1-\frac{1728}{j_{E}}\right)\right)^{1 / 2},
$$

est défini sur une extension de $K$ de degré relatif au plus 2 (pour une détermination quelconque de la racine carrée).

Notons

$$
g_{3}^{\prime}=\left(\frac{8}{27}\left(1-\frac{1728}{j_{E}}\right)\right)^{1 / 2} .
$$


On a

$$
g_{3}^{\prime 2}=\frac{64}{729}-\frac{4096}{27 j_{E}}
$$

d'où on déduit l'estimation (56).

Nous terminons maintenant la démonstration du théorème 2 à partir de l'inégalité (49) démontrée dans le paragraphe précédent.

Quitte à remplacer le corps de base par une extension de degré relatif au plus 2 on peut supposer que la courbe elliptique $E$ a un modèle de Weierstrass comme dans (55). On choisit pour $E^{\sharp}$ le "modèle induit" (voir le lemme 10.2, p. 131 de [Da]), qui est défini sur cette extension de $K$.

Une deuxième extension $K^{\prime}$ de degré relatif au plus Card $\mathrm{GL}_{2}(\mathbb{Z} / 12 \mathbb{Z})=$ 19888 permet de supposer que $\mathbb{P}_{2}\left(K^{\prime}\right)$ contient les points de 12 -torsion de $E$. Ainsi $\left.E\right|_{K}$ est semi-stable, et tous les points de 2-torsion de $E$ sont en particulier définis sur $K^{\prime}$.

Une extension $K^{\prime \prime}$ de degré relatif $3^{2}$ permet enfin de supposer que tous les points de 2-torsion de $E^{\sharp}$ sont définis sur $K^{\prime \prime}$. La quantité $h(E)$ satisfait l'estimation suivante par application des inégalités (50) et (56) :

$$
h(E) \leq 55.65+12 \max \left\{1, h_{\mathcal{F}}(E)\right\} .
$$

On applique l'inégalité (49), où il s'agit de remplacer $d$ par $2 \cdot 3^{2} \cdot 19888 d$. On obtient

$$
\begin{aligned}
N & \leq 1.55 \cdot 10^{68} d^{4}(12.79+\log \max \{e, d\})^{2} \max \left\{1, h_{\mathcal{F}}(E)\right\}^{2} \\
& \leq 10^{75} d^{4}(\log \max \{e, d\})^{2} \max \left\{1, h_{\mathcal{F}}(E)\right\}^{2} .
\end{aligned}
$$

Le théorème 2 est ainsi démontré.

Remerciements. L'auteur remercie S. David pour les innombrables discussions autour de ce texte. Il remercie aussi F. Amoroso, E. Reyssat et M. et A. Waldschmidt pour leur lecture détaillée, et le referee pour son rapport précis.

\section{Références}

[Be] D. Bertrand, Hauteurs et isogénies, dans : Séminaire sur les pinceaux de courbes elliptiques, Astérisque 183 (1990), 107-125.

[Bo] J.-B. Bost, Périodes et isogénies des variétés abéliennes sur les corps de nombres (d'après D. Masser et G. Wüstholz), Astérisque 237 (1996), 115-161.

[Chu] D. V. Chudnovsky and G. V. Chudnovsky, Padé approximations and Diophantine geometry, Proc. Nat. Acad. Sci. U.S.A. 82 (1985), 2212-2216.

[Da] S. David, Minorations de formes linéaires de logarithmes elliptiques, Mém. Soc. Math. France (N.S.) 62 (1995).

[Den] L. Denis, Lemmes de multiplicités et intersections, Comment. Math. Helv. 70 (1995), 235-247. 
[Fa-Ph] A. Faisant et G. Philibert, Quelques résultats de transcendance liés à l'invariant modulaire j, J. Number Theory 25 (1987), 184-200.

[Fal] G. Faltings, Finiteness theorems for abelian varieties over number fields, dans : Arithmetic Geometry, G. Cornell and J. H. Silverman (eds.), traduit de l'original en Allemand, Springer, New York, 1984, 9-27.

[He] O. Herrmann, Über die Berechnung der Fourierkoeffizienten der Funktion $j(\tau)$, J. Reine Angew. Math. 274/275 (1975), 187-195.

[Lan] S. Lang, Elliptic Functions, with an appendix of J. Tate, 2nd ed., Springer, New York, 1987.

[Lau] M. Laurent, Linear forms in two logarithms and interpolation determinants, Acta Arith. 66 (1994), 181-199.

[Ma-Wü1] D. W. Masser and G. Wüstholz, Isogeny estimates for abelian varieties, and finiteness theorems, Ann. of Math. (2) 137 (1993), 459-472.

[Ma-Wü2] -, -, Periods and minimal abelian subvarieties, ibid., 407-458.

[Ma-Wü3] - - - Galois properties of division fields of elliptic curves, Bull. London Math. Soc. 25 (1993), 247-254.

[Ma-Wü4] -, 一, Estimating isogenies on elliptic curves, Invent. Math. 100 (1990), 1-24.

[Pe] F. Pellarin, Application de la méthode de transcendance à deux problèmes d'approximation diophantienne, thèse de doctorat, Univ. P. et M. Curie, 1997.

[Phi1] P. Philippon, Nouveaux lemmes de zéros dans les groupes algébriques commutatifs, Rocky Mountain J. Math. 26 (1996), 1069-1088.

[Phi2] - Lemmes de zéros dans les groupes algébriques, Bull. Soc. Math. France 114 (1986), 355-383.

[Phi-Wa] P. Philippon et M. Waldschmidt, Formes linéaires de logarithmes sur les groupes algébriques commutatifs, Illinois J. Math. 32 (1988), 281-314.

[Se] J.-P. Serre, Abelian $\ell$-Adic Representations and Elliptic Curves, avec la collaboration de W. Kuyk et J. Labute, nouvelle édition révisée de l'original du 1968, A. K. Peters, Wellesley, 1998.

[Sil] J. H. Silverman, Heights and elliptic curves, dans : Arithmetic Geometry, G. Cornell and J. H. Silverman (eds.), Springer, New York, 1984, 253-265.

[Wa1] M. Waldschmidt, A lower bound for linear forms in logarithms, Acta Arith. 37 (1980), 257-283.

[Wa2] -, Linear independence of logarithms of algebraic numbers (avec une appendice par M. Laurent), IMSc Report No. 116, Institute of Mathematical Sciences, Madras, 1992.

Structures Discrètes et Analyse Diophantienne

Université de Caen

Campus II, Boulevard Maréchal Juin

BP 5186

F-14032 Caen Cedex, France

E-mail: pellarin@math.unicaen.fr

Reçu le 15.1.1999

et révisé le 18.1.2001 